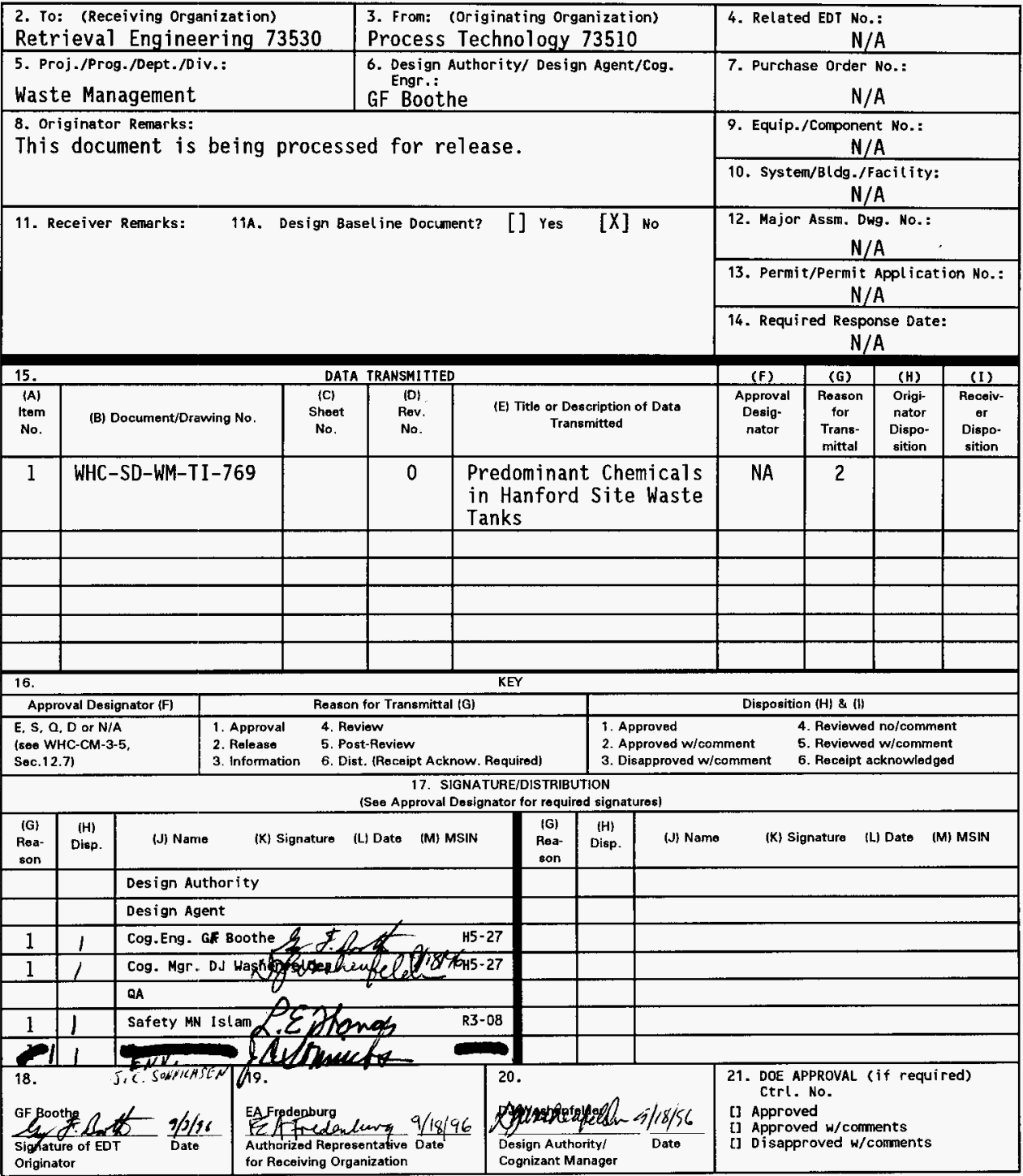

BD-7400-172-2(05/96) GEF097 


\section{Predominant Chemicals in Hanford Site Waste Tanks}

Gary F. Boothe

Westinghouse Hanford Company, Richland, WA 99352

U.S. Department of Energy Contract DE-AC06-87RL10930

$\begin{array}{llll}\text { EDT/ECN: } & 615774 & \text { UC: } & 2000 \\ \text { Org Code: } & 73510 & \text { Charge Code: } & \text { D2086 } \\ \text { B\&R Code: } & \text { EW3130010 } & \text { Total Pages: } & 45\end{array}$

Key Words: chemicals, waste, tanks, hazards

Abstract: Predominant chemical constituents in Hanford Site singleshell and double-shell tanks are determined. Predominant chemical constituents are defined as those anions, cations, and compounds presenting over 99 percent of the routine risks to workers or members of the public. Toxic chemicals and those chemical constituents in tanks that present the 99 percentile hazards to groundwater and air are identified.

TRADEMARK DISCLAIMER. Reference herein to any specific commercial product, process, or service by trade name, trademark, manufacturer, or otherwise, does not necessarily constitute or imply its endorsement, recommendation, or favoring by the United States Goverrment or any agency thereof or its contractors or subcontractors.

Printed in the United States of America. To obtain copies of this document, contact: WHC/BCS Document Control Services, P.O. Box 1970, Mailstop H6-08, Richland WA 99352, Phone (509) 372-2420; Fax (509) 376-4989.
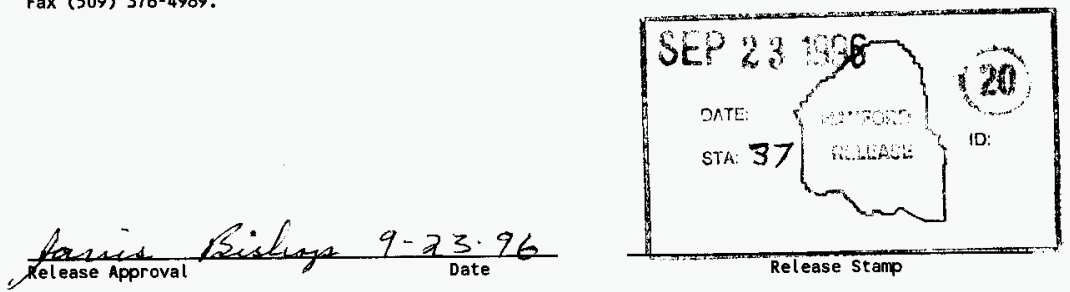

Approved for Public Release 
WHC-SD-WM-TI-769, Rev. 0

\title{
PREDONINANT CHEMICALS IN HANFORD SITE WASTE TANKS
}

\author{
August 1996
}

Gary F. Boothe

Westinghouse Hanford Company

Richland, Washington 
WHC-SD-MM-TI-769, Rev. 0

\section{CONTENTS}

1.0 INTRODUCTION ............................... 1

2.0 PURPOSE OF DOCUMENT .......................... 1

3.0 SCOPE AND LIMITATIONS .................... 1

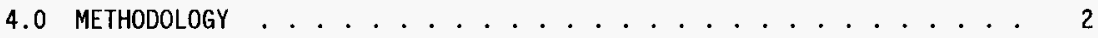

5.0 RESULTS . . . . . . . . . . . . . . . . . . 5

6.0 COMPARISON WITH OTHER STUDIES . . . . . . . . . . . . . 7

7.0 REFERENCES . . . . . . . . . . . . . . . 10

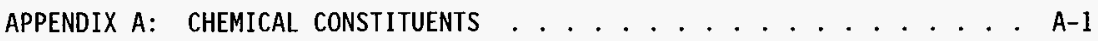

APPENDIX B: CONSTITUENT PREVALENCE RANK ............ B- . . .

APPENDIX $c$ : HAZARDOUS RANK OF CONSTITUENTS ............ c-l

APPENDIX D: MTCA RANK OF CHEMICALS .............. . . . . . 
WHC-SD-WM-TI-769, Rev. 0

Abbreviations and Acronyms

$\begin{array}{ll}\text { ALI } & \text { annual limits on intake } \\ \text { DTPA } & \text { diamine-triamine-pentacetic acid } \\ \text { EDTA } & \text { ethylenediamine-tetracetic acid } \\ \text { HEDTA } & \text { hydroxy-ethylethylene-diamine-triacetic acid } \\ \text { MCL } & \text { maximum contaminant levels } \\ \text { MTCA } & \text { Model Toxics Control Act } \\ \text { PNL } & \text { Pacific Northwest Laboratory } \\ \text { TOC } & \text { total organic carbon }\end{array}$


WHC-SD-WM-TI-769, Rev. 0

\section{PREDOMINANT CHEMICALS IN HANFORD SITE WASTE TANKS}

\subsection{INTRODUCTION}

The chemical inventories of Hanford single-she11 and double-shell waste tanks include a large number of organic and inorganic compounds, anions and cations. The vast majority of tank wastes originated from process chemicals (i.e., chemicals that were used in the processing of nuclear fuels). Fairly reliable information is available on process chemical inventories (Agnew 1996, Shelton 1996). Tank waste also includes a large number and variety of incidental chemicals from supporting plants and operations (Klem 1990). Inventories of these chemicals are not well known. The question addressed by this document is: Out of the hundreds of process and incidental chemical constituents present in waste tanks, which ones are important relative to occupational health and safety and long-term public risk?

\subsection{PURPOSE OF DOCUMENT}

The purpose of this document is to determine the predominant chemical constituents in Hanford Site Waste tanks. By predominant constituents it is meant those chemical species that represent over $99 \%$ of the routine risks to the public or to workers. The predominant constituents are those that should be included in the best-estimate inventory (Kupfer 1996), those that should be considered in environmental impact statements, those that should be considered in facility design and safety evaluations, and those that should be considered relative to tank closure plans.

\subsection{SCOPE AND LIMITATIONS}

The determination of predominant constituents, as defined above, is relevant to both current tank waste inventories and inventories of tank waste residuals after completion of saltwell pumping and sludge washing. The total mass of chemical constituents has no bearing on the determination of predominance, provided that no chemical differentiation is involved in saltwell pumping or sludge washing beyond that differentiation due to solubilities. This is because soluble and insoluble species are ranked in importance separately, and because predominance is strictly determined by the relative ratios of components. 
For purposes of this study it was assumed that saltwell pumping and sludge washing would remove $90 \%$ of the soluble constituents and $50 \%$ of the insoluble constituents from the tanks. The recovered waste would be sent to melters for vitrification and the remaining waste would be the tank residuats. It is recognized that sluicing of the tanks may in fact remove most of the soluble and insoluble constituents (e.g., 99\% removal of waste to achieve Hanford Federal Facility Agreement and Consent Order objectives [Ecology 1993]). However, varying the percentage of waste removal has no impact on the determination of predominance, relative to tank waste residuals or that waste that is sent to the melters for vitrification.

The predominant chemical constituents determined here are based on global inventories. Individual tank inventories may vary considerably from the global inventory, not necessarily in the list of constituents, but certainly in the ratios of constituents. Therefore, there may be additional predominant chemicals on a tank-by-tank basis beyond those identified by this document, or there may be fewer predominant constituents based on tank history. This document provides a starting point in identifying which constituents are of concern in tank waste in general.

This document does not consider accident risks from chemical products or chemical reactions in the tanks. For example, hydrogen generated from radiolysis is not listed as a tank constituent. Fires, explosions and other upset conditions have been addressed in previous safety screening documents and safety analyses.

\subsection{METHODOLOGY}

In an ideal risk ranking of chemical constituents, each constituent would have a water quality standard and an air quality standard, all derived from the same risk basis. Groundwater and airborne hazard indices could then be developed for each constituent by simply dividing the mass of each constituent by its water quality standard and its airborne quality standard. The hazard indices could be modified by transport factors, such as the vadose zone distribution coefficient $\left(K_{d}\right)$, and other factors that account for different degrees of release, transport or concentration in the environment. This type of ranking has been performed for radionuclides, based on ingestion annual limits on intake (ALIs) and inhalation ALIs (Boothe 1996). Unfortunately, it is not possible to perform such a simple ranking for chemical constituents.

An idealized ranking of chemical constituents is not possible because there are no uniform water quality standards or airborne quality standards for all chemical species. Some chemicals have drinking water standards (WHC 1995) such as the maximum contaminant levels (MCLs), and others do not. Some chemicals have Model Toxics Control Act (MTCA) levels (Ecology 1996), and others do not. Some chemicals have no MCLs or MTCAs but may be either toxic or carcinogenic. There are toxicity categories given in hazardous waste regulations, but these have a different bases than drinking water standards. Some chemicals have airborne threshold limits for occupational exposure, and others do not. In any case, airborne standards do not have the same basis as water quality standards. 
In order to determine which chemical constituents are important from a risk standpoint, it is necessary to consider several parameters collectively: the prevalence of the constituent, the toxicity of the constituent or its compounds, the available water quality standards, the listed MTCA values, the available airborne standards, and the mobility and volatility factors.

In this document, four general criteria were developed and employed to determine if a tank waste constituent is predominant:

1. If a soluble constituent (or the constituent's soluble tank compounds) is toxic (i.e., has a toxicity category of $A, B, C, D$, or $X$ in accordance with 10 CFR Part 302 Spi11 Table [EPA 1985]), then the constituent is predominant if global mass concentrations exceed:

1 part per ten million for Category $X$

1 part per one million for Category $A$

1 part per 100 thousand for Category B

1 part per ten thousand for Category $\mathrm{C}$

5 parts per ten thousand for Category $D$

The concentrations listed above are approximately the defining aquatic concentrations for the toxicity categories (EPA 1985). A great deal of effort was made to assign the appropriate and conservative toxicity category to the tank ionic constituents. For example, lead is listed as a tank constituent. Lead compounds are generally toxicity level D; however, for purposes here ionic lead or lead phosphate is conservatively assumed, which is toxicity category $X$. No particular compound is assumed for anions such as chloride, fluoride, hydroxide, nitrate, etc., but the metals are assumed to form compounds with these anions and, consequently, they are covered.

The above criteria does not quantify the risks from the identified toxic materials. Even though a chemical constituent is within a toxicity category based on global concentrations, it does not mean that it is within the 99 percentile risk. However, in the interests of conservatism it is assumed here that together, the toxic materials meeting the above criteria represent some fraction of the 99 percentile risk.

2. If the chemical constituent is within the 99 percentile groundwater hazard based on MCLs, it is considered a predominant constituent. The groundwater hazard is measured by an index $\left(\mathrm{H}_{\mathrm{gw}}\right)$ which is defined by:

$$
\mathrm{H}_{\mathrm{gw}}=\mathrm{M} \times \mathrm{MF} / \mathrm{MCL}_{\mathrm{gw}} \text {, }
$$


where $M$ is the mass of the constituent ( $g$ ), MF is a mobility factor, and $M C L$ is a water quality standard $(\mathrm{g} / \mathrm{L})$ given in the Environmental Protection Manual (WHC 1995). The MF is given by:

$$
\text { MF }=1 /\left(1+5 K_{d}\right) \text {, }
$$

where $K_{d}$ is the distribution coefficient in migrating through the vadose zone to the groundwater. The distribution coefficients were taken from the Tank Waste Remediation System Environmental Impact Statement (DOE 1996).

3. If the chemical constituent is within the 99 percentile groundwater hazard based on MTCAs, it is considered a predominant constituent. The groundwater hazard is measured by an index $\left(H_{g w}\right)$ which is defined by:

$$
H_{g w}=M \times M F / M T C A_{g w} \text {, }
$$

where $M$ is the mass of the constituent (g), MF is a mobility factor, and MTCA is a water quality standard (ug/L) given in the Model Toxics Control Act (Ecology 1996). The MF is given by:

$$
M F=1 /\left(1+5 K_{d}\right) \text {, }
$$

where $K_{d}$ is the distribution coefficient in migrating through the vadose zone to the groundwater. The distribution coefficients were taken from the Tank Waste Remediation System Environmenta1 Impact Statement (DOE 1996).

4. If the chemical constituent is within the 99 percentile airborne hazard, it is considered a predominant constituent. The airborne hazard is measured by an index $\left(H_{a}\right)$ which is defined by:

$$
H_{a}=M \times V F / S_{a} \text {, }
$$

where $M$ is the mass of the constituent (g), VF is a volatility factor, and $S$ is an airborne quality standard $(g / L)$. The airborne quality standard used is the Threshold Limiting Value (ACGIH 1992). The VF is an estimate of the fraction of the constituent that would go airborne under melter conditions, derived from Stegen (1994). No factor for retardation through high-efficiency particulate air filters was used.

Collectively, there is a high level of assurance that the above criteria, applied to the known or bounding inventories assumed, identify the predominant chemical constituents, even though the quantities of specific compounds are not known. Although some constituents have no toxicity category, and some have no MCL, MTCA value, or air quality standard, there are no highly hazardous materials without at least one of these four parameters. 


\subsection{RESULTS}

Appendix A alphabetically lists 140 chemical constituents of interest in Hanford single-shell and double-shell tanks. By chemical constituents of interest it is meant: 1) those chemicals species (anions, cations and specific compounds) that are known to be in the tanks either from waste processing or incidental support operations, and 2) those chemical species that are either major tank waste constituents ( 1 part in 100 million on a global mass basis), or that present aquatic or airborne hazards.

Three separate data bases were used, Agnew (1996), Shelton (1996), and Klem (1990), to derive the chemical constituent list. Agnew lists the major process chemical constituents based on process knowledge, Shelton 1ists major and minor chemical constituents based on historical inventory records, and Klem lists incidental chemical constituents known to have been transferred to tank farms. Many of the constituents from these data bases probably no longer exist in significant quantities in the tanks, having either degraded, evaporated or otherwise escaped from the waste.

Bounding masses of incidental constituents were established from a subjective evaluation of procurement records. Bounding masses of $10 \mathrm{~kg}$ or 100 $\mathrm{kg}$ were assumed, depending on the level of procurement, as indicated by solid waste records (01son 1991). Some constituents were eliminated because they cannot exist in significant quantities in the caustic tank environs (Borsheim and Kirsch 1991). These constituents include ammonium nitrate and acids. Also, it was assumed that those constituents with boiling points less than 100 degrees centigrade would have evaporated from the tanks or would have escaped the waste via the evaporator.

The first section of Appendix A (pages 1 through 6) gives global inventories, water quality standards, soluble fractions, soluble residual masses, $K_{d}$ values, calculated mobility factors, and calculated water hazard indices. The second section of Appendix A (pages 7 through 11) gives insoluble tank residuals, air quality standards, volatility factors, calculated airborne hazard indices and toxicity category. The constituents in bold are toxic chemicals, in accordance with 10 CFR Part 302.

Appendix $B$ is a ranking of soluble tank residuals and melter feed based on prevalence alone. It is assumed that saltwell pumping and sluicing will remove $90 \%$ of the soluble tank inventory and $50 \%$ of the insoluble tank inventory. In applying Criteria 1 above, the predominant toxic soluble constituents are given in Table 1.

Except for ethylenediamine-tetracetic acid (EDTA) and butanol, Table 1 lists no organic compounds other than total organic carbon (TOC). The EDTA is a complexant (along with hydroxy-ethylethylene-diamine-triacetic acid [HEDTA] and diamine-triamine-pentacetic acid [DTPA]). These complexants are actually covered as toxic by total sodium, because they are sodium salts within the tank waste (Agnew 1996). The complexants are also covered as toxic by TOC. However, Table 1 indicates that these organic compounds are especially significant and they should be accounted for separately. 
There are no other specific toxic organic compounds that are of special concern if the global inventory assumptions are valid. This means that specific organic compounds other than EDTA and butanol need not be sampled or analyzed for, since none of them are predominant by themselves.

Table 1. Predominant Toxic Soluble Constituents.

\begin{tabular}{|c|l|l||}
\hline $\begin{array}{c}\text { TOXICITY } \\
\text { CATEGORY }\end{array}$ & \multicolumn{1}{|c|}{$\begin{array}{c}\text { GLOBAL } \\
\text { CONCENTRATION }\end{array}$} & \multicolumn{1}{|c|}{$\begin{array}{c}\text { SOLUBLE } \\
\text { CONSTITUENT }\end{array}$} \\
\hline$x$ & $>.00001 \%$ & $\begin{array}{l}\text { ANTIMONY } \\
\text { CADMIUM } \\
\text { CHROMIUM } \\
\text { COPPER } \\
\text { LEAD } \\
\text { MERCURY } \\
\text { NICKEL } \\
\text { THALLIUM } \\
\text { TOC }\end{array}$ \\
\hline$A$ & $>.0001 \%$ & $\begin{array}{l}\text { CYANIDE } \\
\text { SODIUM }\end{array}$ \\
\hline$B$ & $>.001 \%$ & AMMONIA \\
\hline$C$ & $>.01 \%$ & IRON \\
\hline$D$ & $>.05 \%$ & $\begin{array}{l}\text { BUTANOL } \\
\text { EDTA }\end{array}$ \\
\hline
\end{tabular}

Appendix $C$ is a ranking of chemical constituents in accordance with groundwater hazard and airborne hazard. In accordance with the criteria of items 2 and 3 above, the predominant chemical constituents presenting over $99 \%$ of the groundwater and airborne hazards are:

\begin{tabular}{l} 
GROUNDWATER HAZARD \\
\hline Cadmium \\
Chromium \\
Fluoride \\
Lead \\
Mercury \\
Nitrate \\
Nitrite
\end{tabular}

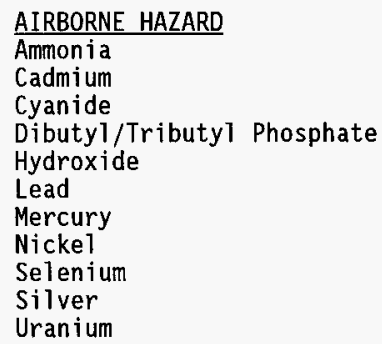

The uranium appears on the above list because of its chemical toxicity, not because it is radioactive. 
Appendix $C$ shows that even if global inventories of incidental chemical constituents are increased by one or two orders of magnitude, there generally would be no change in the predominant constituent list. This is because the hazard index falls off very rapidly after the 99 percentile constituents. For example, the groundwater hazard index for toluene is $1.0 E+7$, which is over 5 orders of magnitude lower than the hazard index of nitrite $(1.35 \mathrm{E}+12)$.

Appendix $D$ ranks chemical constituents in accordance with MTCA groundwater values. The 99 percentile hazardous constituents are:

$\begin{array}{lll}\text { Antimony } & \text { EDTA } & \text { Nitrite } \\ \text { Butanol } & \text { Fluoride } & \text { Sodium } \\ \text { Cadmium } & \text { Hydroxide } & \text { Sulfate } \\ \text { Chloride } & \text { Mercury } & \text { TOC } \\ \text { Chromium } & \text { Nickel } & \text { Uranium } \\ \text { Cyanide } & \text { Nitrate } & \end{array}$

Based on the preceding analyses, the predominant tank waste constituents, representing over $99 \%$ of the routine hazards to occupational and public health and safety, are 1 isted in Table 2. Those chemical constituents that are listed as groundwater hazards, either by the MCL or MTCA ranking, or as toxic should be included in tank closure characterization plans. Other chemical constituents that might become important relative to future waste acceptance criteria or future process requirements may be added to Table 2 from the list of constituents in Appendix A.

\subsection{COMPARISON WITH OTHER STUDIES}

Pacific Northwest Laboratory (PNL) has previously ranked hazardous constituents based on the TRAC data base and vadose zone modeling (PNL 1991). According to this ranking, the greatest nonradiological and noncarcinogenic risks to the groundwater are from:

$\begin{array}{lll}\text { Antimony } & \text { Cyanide } & \text { Nitrate } \\ \text { Beryllium } & \text { EDTA } & \text { Nitrite } \\ \text { Cadmium } & \text { Fluoride } & \text { Silver } \\ \text { Chloride } & \text { Iron } & \text { Sodium } \\ \text { Chromium } & \text { Mercury } & \text { Sulfate } \\ \text { Copper } & \text { Nickel } & \text { Vanadium }\end{array}$

The preceding list is quite similar to the predominant constituent list in Table 2, even though older TRAC data was used in the PNL study (1991), whereas re-normalized TRAC, Agnew (1996) and Klem (1990) were used in this document. The only real differences between the two studies are the appearance of vanadium and beryllium in the PNL list but not the predominant list, and the appearance of TOC, lead, thallium, ammonia, butanol, dibutyl/tributyl phosphate, hydroxide, selenium, and uranium on the predominant 1 ist. 
The appearance of vanadium in the PNL report (1991) is simply inexplicable, since this element's inventory is not 1isted in the PNL report, nor is it listed by Shelton (1996) or Agnew (1996).

The appearance of beryllium in the PNL report is explicable because it is a major constituent in the TRAC data base; however, Appendices $C$ and $D$ show that beryllium is are not within the 99 percentile groundwater or airborne hazard.

The inclusion of TOC, lead, thallium, ammonia, butanol, and hydroxide on the predominant list but not in the PNL study is due to differences in data bases. It should be noted that TOC is conservatively considered to be toxicity category $X$ in this document.

The inclusion of dibutyl/tributyl phosphate and selenium on the predominant list is explicable because these are airborne hazards, which the PNL study does not address. 
Table 2. Predominant Tank Waste Constituents.

\begin{tabular}{|l|l||}
\hline $\begin{array}{c}\text { PREDOMINANT } \\
\text { CANK WASTE } \\
\text { CONSTITUENT }\end{array}$ & \\
\hline AMMONIA & TOXIC COMPOUNDS, AIRBORNE HAZARD \\
\hline ANTIMONY & TOXIC COMPOUNDS, GROUNDWATER HAZARD \\
\hline BUTANOL & TOXIC, GROUNDWATER HAZARD \\
\hline CADMIUM & TOXIC COMPOUNDS, GROUNDWATER HAZARD, AIRBORNE HAZARD \\
\hline CHLORIDE & GROUNDWATER HAZARD \\
\hline CHROMIUM & TOXIC COMPOUNDS, GROUNDWATER HAZARD \\
\hline COPPER & TOXIC COMPOUNDS \\
\hline CYANIDE & TOXIC COMPOUNDS, GROUNDWATER HAZARD, AIRBORNE HAZARD \\
\hline DIBUTYL/TRIBUTYL \\
PHOSPHATE & AIRBORNE HAZARD \\
\hline EDTA & TOXIC, GROUNDWATER HAZARD \\
\hline FLUORIDE & GROUNDWATER HAZARD \\
\hline HYDROXIDE & GROUNDWATER HAZARD, AIRBORNE HAZARD \\
\hline IRON & TOXIC COMPOUNDS \\
\hline LEAD & TOXIC COMPOUNDS, GROUNDWATER HAZARD, AIRBORNE HAZARD \\
\hline MERCURY & TOXIC COMPOUNDS, GROUNDWATER HAZARD, AIRBORNE HAZARD \\
\hline NICKEL & TOXIC COMPOUNDS, GROUNDWATER HAZARD, AIRBORNE HAZARD \\
\hline NITRATE & GROUNDWATER HAZARD \\
\hline NITRITE & GROUNDWATER HAZARD \\
\hline SELENIUM & AIRBORNE HAZARD \\
\hline SILVER & AIRBORNE HAZARD \\
\hline SODIUM & TOXIC COMPOUNDS, GROUNDWATER HAZARD \\
\hline SULFATE & GROUNDWATER HAZARD \\
\hline THALLIUM & TOXIC COMPOUNDS \\
\hline TOC & TOXIC COMPOUNDS, GROUNDWATER HAZARD \\
\hline URANIUM & AIRBORNE HAZARD, GROUNDWATER HAZARD \\
\hline
\end{tabular}




\subsection{REFERENCES}

ACGIH, 1992, Threshold Limit Values for Chemical Substances and Physical Agents and Biological Exposure Indices, American Conference of Governmental Industrial Hygienists, Cincinnati, Ohio.

Agnew, S. F., 1996, Hanford Tank Chemical and Radionuclide inventories: HDW Model Rev. 3, LA-UR-96-858, Los Alamos Nationa1 Laboratory, Los A1 amos, New Mexico.

Boothe, G. F., 1996, Predominant Radionuclides in Hanford Site Waste Tanks, WHC-SD-WM-TI-731, Westinghouse Hanford Company, Richland, Washington.

Borsheim, G. L., Kirch, N. W., 1991, Summary of Single-Shell Tank Waste Stability, WHC-EP-0347, Westinghouse Hanford Company, Richland, Washington.

DOE, 1996, Draft Environmental Impact Statement for the Tank Waste Remediation System, DOE/EIS-0189D, U. S. Department of Energy and Washington State Department of Ecology, Richland, Washington.

Ecology, EPA, and DOE, 1993, Hanford Federal Facility Agreement and Consent Order, as amended, Washington State Department of Ecology, Olympia, Washington, and U.S. Environmental Protection Agency and U.S. Department of Energy, Washington D.C.

Ecology, 1996, Model Toxics Control Act Cleanup Levels and Risk Calculations (CLARC) Update, Washington State Department of Ecology, Publication \#94-145, 01 ympia, Washington.

EPA, 1985, List of Hazardous Substances and Reportable Quantities, 10 CFR 300, U. S. Environmental Protection Agency, Washington, D. C.

Klem, M. J., 1990, Inventory of Chemicals Used at Hanford Site Production Plants and Support Operations (1944-1980), WHC-EP-0172, REV 1, Westinghouse Hanford Company, Richland, Washington.

Kupfer, M. J., 1996, Interim Report: Best-Basis Inventories of Chemica7s and Radionuclides in Hanford Site Tank Waste, WHC-SD-WM-TI-740, Rev. C, Westinghouse Hanford Company, Richland, Washington.

01 son, W. W., 1991, Solid Waste Stream Hazardous and Dangerous Components Study, WHC-SD-WM-RPT-056, Westinghouse Hanford Company, Richland, Washington.

PNL, 1991, Single-She7l Tank Constituent Rankings for Use in Preparing Waste Characterization Plans, PNL-7572, Pacific Northwest Laboratory, Richland, Washington.

Shelton, L. W., 1996, Chemical and Radionuclide Inventory for Single and Double-She17 Tanks, Internal Memo 74A20-96-30, Westinghouse Hanford Company, Richland, Washington. 
WHC-SD-WM-TI-769, Rev, 0

Stegen, G. E., 1994, Volatile and Easily Reduced Components in Melter Feed, DSI194-GES, Westinghouse Hanford Company, Richland, Washington.

WHC, 1993, Environmental Protection Manual, WHC-CM-7-5, Westinghouse Hanford Company, Richland, Washington. 
WHC-SD-WH-TI-769, Rev. 0

APPENDIX A: CHEMICAL CONSTITUENTS

A-1 
SINGLE-SHELL AND DOUBLE-SHELL TANKS

\begin{tabular}{|c|c|c|c|c|c|c|c|c|c|}
\hline & & & & WATER & & TANK & & & \\
\hline & SST & DST & TOTAL & QUALITY & ASSUMED & SOLUBLE & & & WATER \\
\hline TANK WASTE & MASS & MASS & MASS & STANDARD & SOLUBLE & RESIDUALS & ASSUMED & MOBILITY & HAZARD \\
\hline CONSTITUENT(1) & $(\mathrm{kg})(2)$ & $(\mathbf{k g})(2)$ & $(\mathbf{k g})(3)$ & $(\mathrm{mg} / \mathrm{L})(4)$ & FRACTION(5) & $(\mathbf{k g})(5)$ & $\mathrm{Kd}(6)$ & FACTOR(6) & INDEX(7) \\
\hline ACETATE & $5.70 \mathrm{E}+04$ & $4.25 E+04$ & $9.95 E+04$ & 1000 & $1.00 \mathrm{E}+00$ & $9.95 E+03$ & $0.00 \mathrm{E}+00$ & $1.00 \mathrm{E}+00$ & $9.95 \mathrm{E}+06$ \\
\hline ACETONE(12) & & & $0.00 \mathrm{E}+00$ & 1000 & $1.00 E+00$ & $0.00 \mathrm{E}+00$ & $0.00 \mathrm{E}+00$ & $1.00 \mathrm{E}+00$ & $0.00 \mathrm{E}+00$ \\
\hline ALUMINUM & $5.53 E+06$ & $2.19 \mathrm{E}+06$ & $7.72 \mathrm{E}+06$ & 1000 & $0.00 \mathrm{E}+00$ & $0.00 \mathrm{E}+00$ & $5.00 \mathrm{E}+01$ & 3.98E-03 & $0.00 \mathrm{E}+00$ \\
\hline AMMONLA (NH3) & $9.06 \mathrm{E}+04$ & $3.22 \mathrm{E}+05$ & $4.13 E+05$ & 1000 & $1.00 \mathrm{E}+00$ & $4.13 E+04$ & $0.00 E+00$ & $1.00 \mathrm{E}+00$ & $4.13 E+07$ \\
\hline ANTIMONY & & & $1.00 \mathrm{E}+02$ & 0.006 & $1.00 \mathrm{E}+00$ & $1.00 \mathrm{E}+01$ & $0.00 \mathrm{E}+00$ & $1.00 E+00$ & $1.67 \mathrm{E}+09$ \\
\hline ARSENIC & & & $2.30 \mathrm{E}+00$ & 0.05 & $1.80 \mathrm{E}-02$ & $4.14 \mathrm{E}-03$ & $5.00 \mathrm{E}+01$ & $3.98 \mathrm{E}-03$ & $3.30 \mathrm{E}+02$ \\
\hline BARIUM & & & $4.53 E+03$ & 2 & 1.72E-01 & $7.79 \mathrm{E}+01$ & $1.00 \mathrm{E}+01$ & 1.96E-02 & $7.64 \mathrm{E}+05$ \\
\hline BENZENE(12) & & & $0.00 \mathrm{E}+00$ & 0.005 & $1.00 \mathrm{E}+00$ & $0.00 \mathrm{E}+00$ & $0.00 \mathrm{E}+00$ & $1.00 \mathrm{E}+00$ & $0.00 \mathrm{E}+00$ \\
\hline BERYLIUM & & & $3.10 \mathrm{E}+00$ & 0.004 & $8.00 \mathrm{E}-03$ & $2.48 \mathrm{E}-03$ & $5.00 \mathrm{E}+01$ & $3.98 \mathrm{E}-03$ & $2.47 \mathrm{E}+03$ \\
\hline BICARBONATE & & & $1.00 \mathrm{E}+02$ & 1000 & $1.00 \mathrm{E}+00$ & $1.00 \mathrm{E}+01$ & $1.00 E+00$ & 1.67E-01 & 1.67E+03 \\
\hline BISMUTH & $5.13 E+05$ & $1.13 E+04$ & $5.24 \mathrm{E}+05$ & 1000 & 4.50E-02 & $2.36 E+03$ & 1.00E+00 & 1.67E-01 & $3.93 E+05$ \\
\hline BISULFATE (AMMONIUM) & & & $1.00 E+02$ & 1000 & $1.00 \mathrm{E}+00$ & $1.00 \mathrm{E}+01$ & $0.00 \mathrm{E}+00$ & $1.00 \mathrm{E}+00$ & $1.00 \mathrm{E}+04$ \\
\hline BISULFITE & & & 1.00E+02 & 1000 & $1.00 \mathrm{E}+00$ & $1.00 \mathrm{E}+01$ & $0.00 \mathrm{E}+00$ & $1.00 \mathrm{E}+00$ & $1.00 \mathrm{E}+04$ \\
\hline BORON & & & $3.43 E+01$ & 1000 & $1.00 \mathrm{E}+00$ & $3.43 \mathrm{E}+00$ & $5.00 \mathrm{E}+01$ & 3.98E-03 & $1.37 \mathrm{E}+01$ \\
\hline BROMATE & & & 1.00E+02 & 1000 & $1.00 \mathrm{E}+00$ & $1.00 \mathrm{E}+01$ & $0.00 \mathrm{E}+00$ & $1.00 \mathrm{E}+00$ & $1.00 \mathrm{E}+04$ \\
\hline BROMINE & & & $1.00 \mathrm{E}+02$ & 1000 & $1.00 \mathrm{E}+00$ & $1.00 \mathrm{E}+01$ & $0.00 \mathrm{E}+00$ & $1.00 \mathrm{E}+00$ & $1.00 \mathrm{E}+04$ \\
\hline BROMOCRESOL PURPLE & & & $1.00 \mathrm{E}+02$ & 1000 & $1.00 \mathrm{E}+00$ & $1.00 \mathrm{E}+01$ & $0.00 \mathrm{E}+00$ & $1.00 E+00$ & $1.00 \mathrm{E}+04$ \\
\hline BROMONAPHTHALENE & & & $1.00 \mathrm{E}+02$ & 1000 & $1.00 \mathrm{E}+00$ & $1.00 \mathrm{E}+01$ & $0.00 \mathrm{E}+00$ & $1.00 E+00$ & $1.00 \mathrm{E}+04$ \\
\hline BUTANOL (BUTYL ALCOHOL) & $1.15 E+05$ & $8.34 E+04$ & $1.98 \mathrm{E}+05$ & 1000 & $1.00 \mathrm{E}+00$ & $1.98 \mathrm{E}+04$ & $0.00 \mathrm{E}+00$ & $1.00 \mathrm{E}+00$ & $1.98 \mathrm{E}+07$ \\
\hline C6H5O7 & $3.72 E+05$ & $3.06 \mathrm{E}+05$ & $6.78 \mathrm{E}+05$ & 1000 & $1.00 \mathrm{E}+00$ & $6.78 E+04$ & $0.00 \mathrm{E}+00$ & $1.00 E+00$ & $6.78 \mathrm{E}+07$ \\
\hline CADMIUM & & & $1.42 E+04$ & 0.005 & $5.38 \mathrm{E}-01$ & $7.64 E+02$ & $1.00 \mathrm{E}+00$ & 1.67E-01 & $2.55 \mathrm{E}+10$ \\
\hline CALCIUM & 4.97E+05 & $1.22 \mathrm{E}+05$ & $6.19 \mathrm{E}+05$ & 1000 & $1.02 \mathrm{E}-01$ & $6.31 E+03$ & $1.00 \mathrm{E}+00$ & 1.67E-01 & $1.05 E+06$ \\
\hline CANCRINITE & & & $2.70 \mathrm{E}+06$ & 1000 & $0.00 \mathrm{E}+00$ & $0.00 E+00$ & 5.00E+01 & $3.98 \mathrm{E}-03$ & $0.00 E+00$ \\
\hline CARBIDE (BORON) & & & $1.00 E+02$ & 1000 & $0.00 \mathrm{E}+00$ & $0.00 \mathrm{E}+00$ & $5.00 \mathrm{E}+01$ & $3.98 \mathrm{E}-03$ & $0.00 \mathrm{E}+00$ \\
\hline CARBON TETRACHLORIDE(12) & & & $0.00 \mathrm{E}+00$ & 0.005 & $1.00 \mathrm{E}+00$ & $0.00 \mathrm{E}+00$ & $0.00 \mathrm{E}+00$ & $1.00 \mathrm{E}+00$ & $0.00 \mathrm{E}+00$ \\
\hline CARBONATE & $2.88 \mathrm{E}+06$ & $1.95 E+06$ & $4.83 E+06$ & 1000 & 9.66E-01 & $4.67 E+05$ & $5.00 \mathrm{E}+01$ & 3.98E-03 & $1.86 \mathrm{E}+06$ \\
\hline CERIUM & & & $2.37 E+05$ & 1000 & $1.00 \mathrm{E}-02$ & 2.37E+02 & $5.00 E+01$ & $3.98 \mathrm{E}-03$ & $9.44 E+02$ \\
\hline CESIUM & & & $2.09 E+03$ & 1000 & 9.01E-01 & $1.88 \mathrm{E}+02$ & $5.00 E+01$ & $3.98 \mathrm{E}-03$ & $7.50 \mathrm{E}+02$ \\
\hline CHLORIDE & $5.76 E+05$ & $3.81 \mathrm{E}+05$ & $9.57 E+05$ & 250 & 9.87E-01 & $9.45 E+04$ & $0.00 \mathrm{E}+00$ & $1.00 \mathrm{E}+00$ & $3.78 \mathrm{E}+08$ \\
\hline CHROMIUM & $6.52 E+05$ & $1.33 E+05$ & $7.85 \mathrm{E}+05$ & 0.1 & $7.40 \mathrm{E}-01$ & $5.81 \mathrm{E}+04$ & $0.00 \mathrm{E}+00$ & $1.00 \mathrm{E}+00$ & $5.81 E+11$ \\
\hline COBALT & & & $4.23 \mathrm{E}+01$ & 1000 & $0.00 \mathrm{E}+00$ & $0.00 E+00$ & $5.00 \mathrm{E}+01$ & 3.98E-03 & $0.00 E+00$ \\
\hline COPPER & & & $2.87 \mathrm{E}+02$ & 1000 & 3.44E-01 & $9.87 \mathrm{E}+00$ & $5.00 \mathrm{E}+01$ & $3.98 \mathrm{E}-03$ & $3.93 E+01$ \\
\hline CYANIDE & $1.44 E+05$ & $0.00 E+00$ & $1.44 \mathrm{E}+05$ & 1000 & $1.00 \mathrm{E}+00$ & $1.44 E+04$ & $0.00 \mathrm{E}+00$ & $1.00 \mathrm{E}+00$ & $1.44 E+07$ \\
\hline
\end{tabular}


WHC-SD-WM-TI-769

CHEMICAL CONSTITUENTS

SINGLE-SHELL AND DOUBLE-SHELL TANKS

\begin{tabular}{|c|c|c|c|c|c|c|c|c|c|}
\hline & & & & WATER & & TANK & & & \\
\hline & SST & DST & TOTAL & QUALITY & ASSUMED & SOLUBLE & & & WATER \\
\hline TANK WASTE & MASS & MASS & MASS & STANDARD & SOLUBLE & RESIDUALS & ASSUMED & MOBILITY & HAZARD \\
\hline CONSTITUENT(1) & $(\mathbf{k g})(2)$ & $(\mathbf{k g})(2)$ & $(\mathbf{k g})(3)$ & $(\mathrm{mg} / \mathrm{L})(\mathbf{4})$ & FRACTION(5) & $(\mathbf{k g})(5)$ & $\mathrm{Kd}(6)$ & FACTOR(6) & INDEX(7) \\
\hline DIATOMACEOUS EARTH & & & $1.00 \mathrm{E}+05$ & 1000 & $0.00 \mathrm{E}+00$ & $0.00 E+00$ & $5.00 \mathrm{E}+01$ & $3.98 \mathrm{E}-03$ & $0.00 \mathrm{E}+00$ \\
\hline DIBUTYL PHOSPHANATE & & & $1.00 E+02$ & 1000 & $1.00 \mathrm{E}+00$ & $1.00 E+01$ & $0.00 \mathrm{E}+00$ & $1.00 \mathrm{E}+00$ & $1.00 \mathrm{E}+04$ \\
\hline DIBUTYLTRIBUTYL PHOSPHATE & $4.12 E+05$ & $1.82 \mathrm{E}+05$ & $5.94 E+05$ & 1000 & $1.00 \mathrm{E}+00$ & $5.94 E+04$ & $0.00 \mathrm{E}+00$ & $1.00 \mathrm{E}+00$ & $5.94 E+07$ \\
\hline DICHROMATE & & & $1.00 E+02$ & 1000 & $0.00 \mathrm{E}+00$ & $0.00 \mathrm{E}+00$ & $5.00 E+01$ & $3.98 \mathrm{E}-03$ & $0.00 \mathrm{E}+00$ \\
\hline DIHYDROXYANTHRAQUINONE(12) & & & $0.00 \mathrm{E}+00$ & 1000 & $1.00 \mathrm{E}+00$ & $0.00 \mathrm{E}+00$ & $0.00 \mathrm{E}+00$ & $1.00 \mathrm{E}+00$ & $0.00 \mathrm{E}+00$ \\
\hline DIOXANE(12) & & & $0.00 E+00$ & 7 & $1.00 E+00$ & $0.00 \mathrm{E}+00$ & $0.00 E+00$ & $1.00 \mathrm{E}+00$ & $0.00 E+00$ \\
\hline DTPA & & & $1.00 \mathrm{E}+02$ & 1000 & $1.00 E+00$ & $1.00 E+01$ & $0.00 E+00$ & $1.00 E+00$ & $1.00 E+04$ \\
\hline DUODECANE & & & $1.00 \mathrm{E}+02$ & 1000 & $1.00 \mathrm{E}+00$ & $1.00 \mathrm{E}+01$ & $0.00 E+00$ & $1.00 E+00$ & $1.00 \mathrm{E}+04$ \\
\hline EDTA & $2.95 E+05$ & $3.24 E+05$ & $6.19 \mathrm{E}+05$ & 1000 & $1.00 \mathrm{E}+00$ & $6.19 \mathrm{E}+04$ & $0.00 \mathrm{E}+00$ & $1.00 \mathrm{E}+00$ & $6.19 E+07$ \\
\hline ETHANOL & & & $1.00 \mathrm{E}+02$ & 1000 & $1.00 \mathrm{E}+00$ & $1.00 \mathrm{E}+01$ & $0.00 E+00$ & $1.00 \mathrm{E}+00$ & $1.00 E+04$ \\
\hline ETHYL ETHER(12) & & & $0.00 E+00$ & 1000 & $1.00 \mathrm{E}+00$ & $0.00 \mathrm{E}+00$ & $0.00 E+00$ & $1.00 \mathrm{E}+00$ & $0.00 E+00$ \\
\hline ETHYLENE GLYCOL & & & $1.00 \mathrm{E}+02$ & 1000 & $1.00 \mathrm{E}+00$ & $1.00 E+01$ & $0.00 E+00$ & $1.00 \mathrm{E}+00$ & $1.00 E+04$ \\
\hline FERROUS SULFAMATE & & & $1.00 E+03$ & 1000 & $1.00 \mathrm{E}+00$ & $1.00 \mathrm{E}+02$ & $0.00 \mathrm{E}+00$ & $1.00 \mathrm{E}+00$ & $1.00 \mathrm{E}+05$ \\
\hline FLUORIDE & & & $1.21 \mathrm{E}+06$ & 4 & $9.43 \mathrm{E}-01$ & $1.14 \mathrm{E}+05$ & $0.00 E+00$ & $1.00 \mathrm{E}+00$ & $2.85 E+10$ \\
\hline FLUOROSILICATE (AMMONIUM) & & & $1.00 \mathrm{E}+02$ & 1000 & $1.00 \mathrm{E}+00$ & $1.00 \mathrm{E}+01$ & $0.00 E+00$ & $1.00 \mathrm{E}+00$ & $1.00 \mathrm{E}+04$ \\
\hline FORMALDEHYDE(12) & & & $0.00 \mathrm{E}+00$ & 1000 & $1.00 \mathrm{E}+00$ & $0.00 \mathrm{E}+00$ & $0.00 E+00$ & $1.00 \mathrm{E}+00$ & $0.00 \mathrm{E}+00$ \\
\hline GALLIUM & & & $1.00 E+02$ & 1000 & $1.00 \mathrm{E}+00$ & $1.00 \mathrm{E}+01$ & $5.00 \mathrm{E}+01$ & $3.98 \mathrm{E}-03$ & $3.98 E+01$ \\
\hline GLUCONATE (SODIUM) & & & $1.00 \mathrm{E}+02$ & 1000 & $1.00 E+00$ & $1.00 \mathrm{E}+01$ & $0.00 \mathrm{E}+00$ & $1.00 \mathrm{E}+00$ & $1.00 \mathrm{E}+04$ \\
\hline GLYCEROL & & & $1.00 E+02$ & 1000 & $1.00 \mathrm{E}+00$ & $1.00 \mathrm{E}+01$ & $0.00 E+00$ & $1.00 \mathrm{E}+00$ & $1.00 \mathrm{E}+04$ \\
\hline GLYCOLATE & $4.07 E+05$ & $6.93 \mathrm{E}+05$ & $1.10 E+06$ & 1000 & $1.00 \mathrm{E}+00$ & $1.10 \mathrm{E}+05$ & $0.00 \mathrm{E}+00$ & $1.00 \mathrm{E}+00$ & $1.10 \mathrm{E}+08$ \\
\hline GOLD & & & $0.00 \mathrm{E}+00$ & 1000 & $0.00 \mathrm{E}+00$ & $0.00 \mathrm{E}+00$ & $5.00 \mathrm{E}+01$ & $3.98 \mathrm{E}-03$ & $0.00 \mathrm{E}+00$ \\
\hline HEDTA & $4.79 E+05$ & $5.55 \mathrm{E}+05$ & $1.03 \bar{E}+06$ & 1000 & $1.00 \mathrm{E}+00$ & $1.03 \mathrm{E}+05$ & $0.00 \mathrm{E}+00$ & $1.00 \mathrm{E}+00$ & $1.03 E+08$ \\
\hline HYDRAZINE(12) & & & $0.00 E+00$ & 0.03 & $1.00 \mathrm{E}+00$ & $0.00 E+00$ & $0.00 E+00$ & $1.00 \mathrm{E}+00$ & $0.00 \mathrm{E}+00$ \\
\hline HYDROGEN SULFIDE(12) & & & $0.00 \mathrm{E}+00$ & 1000 & $1.00 \mathrm{E}+00$ & $0.00 \mathrm{E}+00$ & $0.00 E+00$ & $1.00 E+00$ & $0.00 \mathrm{E}+00$ \\
\hline HYOROXIDE & & & $1.99 \mathrm{E}+07$ & 1000 & $7.18 \mathrm{E}-01$ & $1.43 E+06$ & $0.00 E+00$ & $1.00 \mathrm{E}+00$ & $1.43 \mathrm{E}+09$ \\
\hline HYDROXYLAMINE (NITRATE) & & & $1.00 E+02$ & 1000 & $1.00 \mathrm{E}+00$ & $1.00 \mathrm{E}+01$ & $0.00 \mathrm{E}+00$ & $1.00 \mathrm{E}+00$ & $1.00 \mathrm{E}+04$ \\
\hline HYDROXYLAMINE HYDR.(12) & & & $0.00 \mathrm{E}+00$ & 1000 & $1.00 \mathrm{E}+00$ & $0.00 E+00$ & $0.00 E+00$ & $1.00 \mathrm{E}+00$ & $0.00 \mathrm{E}+00$ \\
\hline HYDROXYQUINOLINE(12) & & & $0.00 \mathrm{E}+00$ & 1000 & $1.00 \mathrm{E}+00$ & $0.00 \mathrm{E}+00$ & $0.00 \mathrm{E}+00$ & $1.00 \mathrm{E}+00$ & $0.00 E+00$ \\
\hline HYPOCHLORITE (SODIUM) & & & $1.00 E+01$ & 1000 & $1.00 \mathrm{E}+00$ & $1.00 E+00$ & $0.00 E+00$ & $1.00 \mathrm{E}+00$ & $1.00 E+03$ \\
\hline IODINE(12) & & & $0.00 E+00$ & 1000 & $1.00 \mathrm{E}+00$ & $0.00 \mathrm{E}+00$ & $0.00 E+00$ & $1.00 \mathrm{E}+00$ & $0.00 \mathrm{E}+00$ \\
\hline IRON & $1.61 E+06$ & $2.20 E+05$ & $1.83 E+06$ & 0.3 & $4.00 \mathrm{E}-02$ & $7.32 E+03$ & $1.00 E+00$ & 1.67E-01 & $4.07 E+09$ \\
\hline ISOAMYL ACETATE & & & $1.00 \mathrm{E}+02$ & 1000 & $1.00 \mathrm{E}+00$ & $1.00 \mathrm{E}+01$ & $0.00 E+00$ & $1.00 \mathrm{E}+00$ & $1.00 E+04$ \\
\hline ISOPROPYL ALCOHOL(12) & & & $0.00 \mathrm{E}+00$ & 1000 & $1.00 E+00$ & $0.00 E+00$ & $0.00 E+00$ & $1.00 \mathrm{E}+00$ & $0.00 E+00$ \\
\hline
\end{tabular}


WHC-SD-WM-TI-769

CHEMICAL CONSTITUENTS

SINGLE-SHELL AND DOUBLE-SHELL TANKS

\begin{tabular}{|c|c|c|c|c|c|c|c|c|c|}
\hline & & & & WATER & & TANK & & & \\
\hline & SST & DST & TOTAL & QUALITY & ASSUMED & SOLUBLE & & & WATER \\
\hline TANK WASTE & MASS & MASS & MASS & STANDARD & SOLUBLE & RESIDUALS & ASSUMED & MOBILITY & HAZARD \\
\hline CONSTITUENT(1) & $(\mathrm{kg})(2)$ & $(\mathrm{kg})(2)$ & $(\mathbf{k g})(3)$ & $(\mathrm{mg} / \mathrm{L})(4)$ & FRACTION(5) & $(\mathbf{k g})(5)$ & $\mathbf{K d}(6)$ & FACTOR(6) & INDEX(7) \\
\hline KEROSENE & & & $1.00 \mathrm{E}+02$ & 1000 & $1.00 \mathrm{E}+00$ & $1.00 \mathrm{E}+01$ & $0.00 \mathrm{E}+00$ & $1.00 \mathrm{E}+00$ & $1.00 \mathrm{E}+04$ \\
\hline LANTHANUM & $4.03 \mathrm{E}+04$ & $1.40 E+02$ & $4.04 E+04$ & 1000 & $1.00 \mathrm{E}-02$ & $4.04 \mathrm{E}+01$ & $5.00 \mathrm{E}+01$ & 3.98E-03 & $1.61 \mathrm{E}+02$ \\
\hline LEAD & $2.68 \mathrm{E}+05$ & $1.07 \mathrm{E}+04$ & $2.79 E+05$ & 0.002 & 2.47E-01 & $6.88 \mathrm{E}+03$ & $1.00 \mathrm{E}+01$ & 1.96E-02 & $6.75 E+10$ \\
\hline LITHIUM & & & $1.00 \mathrm{E}+02$ & 1000 & $1.00 \mathrm{E}+00$ & $1.00 \mathrm{E}+01$ & $0.00 \mathrm{E}+00$ & $1.00 \mathrm{E}+00$ & $1.00 \mathrm{E}+04$ \\
\hline MAGNESIUM & & & $8.25 \bar{E}+03$ & 1000 & $0.00 E+00$ & $0.00 E+00$ & $5.00 \mathrm{E}+01$ & 3.98E-03 & $0.00 E+00$ \\
\hline MANGANESE & & & $1.90 E+05$ & 0.05 & $5.70 \mathrm{E}-02$ & $1.08 \mathrm{E}+03$ & $1.00 \mathrm{E}+01$ & 1.96E-02 & $4.25 E+08$ \\
\hline MERCURY & $5.81 E+03$ & $1.05 E+03$ & $6.86 E+03$ & 0.002 & $3.98 \mathrm{E}-01$ & $2.73 E+02$ & $0.00 \mathrm{E}+00$ & $1.00 E+00$ & $1.37 E+11$ \\
\hline METHANOL(12) & & & $0.00 E+00$ & 1000 & $1.00 E+00$ & $0.00 E+00$ & $0.00 \mathrm{E}+00$ & $1.00 E+00$ & $0.00 \mathrm{E}+00$ \\
\hline METHYL ETHYL KEYTONE(12) & & & $0.00 \mathrm{E}+00$ & 1000 & $1.00 E+00$ & $0.00 E+00$ & $0.00 \mathrm{E}+00$ & $1.00 E+00$ & $0.00 E+00$ \\
\hline METHYL ISOBUTYL KETONE(12) & & & $0.00 \mathrm{E}+00$ & 1000 & $1.00 E+00$ & $0.00 E+00$ & $0.00 \mathrm{E}+00$ & $1.00 \mathrm{E}+00$ & $0.00 E+00$ \\
\hline MOLYBDENUM & & & $3.94 \mathrm{E}+01$ & 1000 & $1.83 E-01$ & 7.21E-01 & $5.00 \mathrm{E}+01$ & $3.98 \mathrm{E}-03$ & $2.87 E+00$ \\
\hline MORPHOLINE & & & $1.00 E+02$ & 1000 & 1.00E+00 & $1.00 \mathrm{E}+01$ & $0.00 \mathrm{E}+00$ & $1.00 \mathrm{E}+00$ & $1.00 \mathrm{E}+04$ \\
\hline NAPHTHYLAMINE & & & $1.00 \mathrm{E}+01$ & 1000 & $1.00 \mathrm{E}+00$ & $1.00 \mathrm{E}+00$ & $0.00 E+00$ & $1.00 \mathrm{E}+00$ & $1.00 \mathrm{E}+03$ \\
\hline NEODYMIUM & & & $1.00 E+02$ & 1000 & $0.00 E+00$ & $0.00 \mathrm{E}+00$ & $5.00 \mathrm{E}+01$ & $3.98 \mathrm{E}-03$ & $0.00 \mathrm{E}+00$ \\
\hline NICKEL & & & $2.14 \mathrm{E}+05$ & 1000 & $3.80 \mathrm{E}-02$ & $8.13 E+02$ & $1.00 \mathrm{E}+00$ & 1.67E-01 & $1.36 \mathrm{E}+05$ \\
\hline NIOBIUM & & & $1.00 E+02$ & 1000 & $1.00 \mathrm{E}+00$ & $1.00 \mathrm{E}+01$ & $5.00 \mathrm{E}+01$ & $3.98 \mathrm{E}-03$ & $3.98 \mathrm{E}+01$ \\
\hline NITRATE & & & $1.08 \mathrm{E}+08$ & 10 & 9.91E-01 & 1.07E+07 & $0.00 \mathrm{E}+00$ & $1.00 \mathrm{E}+00$ & $1.07 E+12$ \\
\hline NITRITE & $8.98 E+06$ & $4.58 \mathrm{E}+06$ & $1.36 \mathrm{E}+07$ & 1 & 9.95E-01 & $1.35 \mathrm{E}+06$ & $0.00 \mathrm{E}+00$ & $1.00 \mathrm{E}+00$ & $1.35 E+12$ \\
\hline OPHENATROLINE & & & $1.00 \mathrm{E}+02$ & 1000 & $1.00 \mathrm{E}+00$ & $1.00 \mathrm{E}+01$ & $0.00 \mathrm{E}+00$ & $1.00 \mathrm{E}+00$ & $1.00 \mathrm{E}+04$ \\
\hline OXALATE (SODIUM) & $6.91 \mathrm{E}+04$ & $7.40 \mathrm{E}+01$ & $6.92 \mathrm{E}+04$ & 1000 & $1.00 \mathrm{E}+00$ & $6.92 \mathrm{E}+03$ & $0.00 \mathrm{E}+00$ & $1.00 \mathrm{E}+00$ & $6.92 \mathrm{E}+06$ \\
\hline PARAFFIN (NORMAL) HYDROCAR. & & & $1.00 E+02$ & 1000 & $0.00 E+00$ & $0.00 \mathrm{E}+00$ & $5.00 \mathrm{E}+01$ & $3.98 \mathrm{E}-03$ & $0.00 \mathrm{E}+00$ \\
\hline PERMANGANATE (POTASSIUM) & & & $1.00 E+02$ & 1000 & $1.00 E+00$ & $1.00 E+01$ & $0.00 E+00$ & $1.00 \mathrm{E}+00$ & $1.00 \mathrm{E}+04$ \\
\hline PERSULFATE (POTASSIUM) & & & $1.00 \mathrm{E}+02$ & 1000 & $1.00 \mathrm{E}+00$ & $1.00 \mathrm{E}+01$ & $0.00 \mathrm{E}+00$ & $1.00 \mathrm{E}+00$ & $1.00 \mathrm{E}+04$ \\
\hline PERSULFATE (SODIUM) & & & $1.00 \mathrm{E}+02$ & 1000 & $1.00 \mathrm{E}+00$ & $1.00 \mathrm{E}+01$ & $0.00 \mathrm{E}+00$ & $1.00 \mathrm{E}+00$ & $1.00 \mathrm{E}+04$ \\
\hline PHOSPHATE & $3.31 \mathrm{E}+06$ & $7.15 \mathrm{E}+05$ & $4.03 E+06$ & 1000 & $6.33 \mathrm{E}-01$ & $2.55 \mathrm{E}+05$ & $1.00 \mathrm{E}+01$ & $1.96 \mathrm{E}-02$ & $5.00 \mathrm{E}+06$ \\
\hline PHOSPHORUS PENTOXIDE & & & $1.00 \mathrm{E}+02$ & 1000 & $1.00 \mathrm{E}+00$ & $1.00 \mathrm{E}+01$ & $0.00 \mathrm{E}+00$ & $1.00 \mathrm{E}+00$ & $1.00 \mathrm{E}+04$ \\
\hline PLATINUM & & & $0.00 E+00$ & 1000 & $0.00 \mathrm{E}+00$ & $0.00 E+00$ & $5.00 \mathrm{E}+01$ & $3.98 \mathrm{E}-03$ & $0.00 E+00$ \\
\hline POTASSIUM & & & $7.36 E+05$ & 1000 & $9.60 \mathrm{E}-01$ & $7.07 E+04$ & $0.00 \mathrm{E}+00$ & $1.00 \mathrm{E}+00$ & $7.07 \mathrm{E}+07$ \\
\hline PRASEODYMIUM & & & $1.00 \mathrm{E}+02$ & 1000 & $0.00 \mathrm{E}+00$ & $0.00 \mathrm{E}+00$ & $5.00 \mathrm{E}+01$ & 3.98E-03 & $0.00 \mathrm{E}+00$ \\
\hline RHENIUM & & & 9.73E-02 & 1000 & $0.00 \mathrm{E}+00$ & $0.00 \mathrm{E}+00$ & $5.00 \mathrm{E}+01$ & 3.98E-03 & $0.00 \mathrm{E}+00$ \\
\hline RHODIUM & & & $1.94 \mathrm{E}+02$ & 1000 & $1.00 \mathrm{E}+00$ & $1.94 \mathrm{E}+01$ & $0.00 E+00$ & $1.00 \mathrm{E}+00$ & $1.94 \mathrm{E}+04$ \\
\hline RUBIDIUM & & & 1.17E+01 & 1000 & $0.00 \mathrm{E}+00$ & $0.00 \mathrm{E}+00$ & $5.00 \mathrm{E}+01$ & $3.98 \mathrm{E}-03$ & $0.00 \mathrm{E}+00$ \\
\hline RUTHENIUM & & & 1.17E+00 & 1000 & $1.00 \mathrm{E}+00$ & 1.17E-01 & $0.00 \mathrm{E}+00$ & $1.00 \mathrm{E}+00$ & $1.17 \mathrm{E}+02$ \\
\hline
\end{tabular}


WHC-SD-WM-TI-769

CHEMICAL CONSTITUENTS

SINGLE-SHELL AND DOUBLE-SHELL TANKS

\begin{tabular}{|c|c|c|c|c|c|c|c|c|c|}
\hline & & & & & & & & & \\
\hline & SST & DST & & & & TANK & & & \\
\hline TANK WASTE & MASS & Dol & TOTAL & QUALITY & ASSUMED & SOLUBLE & & & WATER \\
\hline CONSTITUENT(1) & MASS & MASS & MASS & STANDARD & SOLÜBLE & RESIDUALS & ASSUMED & MOBILITY & HAZARD \\
\hline SDIPHENYL CARBAZIDE & $(\mathrm{kg})(2)$ & $(\mathrm{kg})(2)$ & $(\mathrm{kg})(3)$ & $(\mathrm{mg} / \mathrm{L})(4)$ & FRACTION(5) & $(\mathrm{kg})(5)$ & $K d(6)$ & FACTOR(6) & INDEX $(\overline{7})$ \\
\hline $\begin{array}{l}\text { SDIPHENYL CARBAZIDE } \\
\text { SELENIUM }\end{array}$ & & & $1.00 \mathrm{E}+02$ & 1000 & $0.00 \mathrm{E}+00$ & $0.00 \mathrm{E}+00$ & $0.00 \mathrm{E}+00$ & $1.00 \mathrm{E}+00$ & $0.00 E+00$ \\
\hline & & & $3.31 E+03$ & 0.05 & $0.00 \mathrm{E}+00$ & $0.00 \mathrm{E}+00$ & $0.00 \mathrm{E}+00$ & $1.00 \mathrm{E}+00$ & $0.00 E+00$ \\
\hline $\begin{array}{l}\text { SILICON (SILICATE) } \\
\text { SII VER }\end{array}$ & 4.77E+05 & $1.36 \mathrm{E}+05$ & $6.13 E+05$ & 1000 & $6.40 \mathrm{E}-02$ & $3.92 E+03$ & $5.00 \mathrm{E}+01$ & $3.98 \mathrm{E}-03$ & $1.56 \mathrm{E}+04$ \\
\hline SIIVER & & & $1.80 \mathrm{E}+03$ & 1000 & $1.78 \mathrm{E}-01$ & $3.20 \mathrm{E}+01$ & $5.00 \mathrm{E}+01$ & $3.98 \mathrm{E}-03$ & $1.28 \mathrm{E}+02$ \\
\hline SODIUM & & & $6.88 E+07$ & 1000 & $9.88 \mathrm{E}-01$ & $6.80 \mathrm{E}+06$ & $0.00 E+00$ & $1.00 \mathrm{E}+00$ & $6.80 \mathrm{E}+09$ \\
\hline STRONTIUM & & & $3.69 \mathrm{E}+04$ & 1000 & $1.00 E-02$ & $3.69 \mathrm{E}+01$ & $1.00 \mathrm{E}+01$ & 1.96E-02 & $7.24 E+02$ \\
\hline STYRENE & & & $1.00 \mathrm{E}+01$ & 1000 & $1.00 E+00$ & $1.00 \mathrm{E}+00$ & $0.00 \mathrm{E}+00$ & $1.00 \mathrm{E}+00$ & $1.00 \mathrm{E}+03$ \\
\hline SUGAR & & & $1.00 \bar{E}+05$ & 1000 & $1.00 \mathrm{E}+00$ & $1.00 \mathrm{E}+04$ & $0.00 \mathrm{E}+00$ & $1.00 \mathrm{E}+00$ & $1.00 \mathrm{E}+07$ \\
\hline SULFATE & $2.00 \mathrm{E}+06$ & $1.22 E+06$ & $3.22 E+06$ & 250 & 9.87E-01 & $3.18 \mathrm{E}+05$ & $0.00 E+00$ & $1.00 \mathrm{E}+00$ & $1.27 \mathrm{E}+09$ \\
\hline SULFITE (AMMONIUM) & & & $1.00 \mathrm{E}+02$ & 1000 & $1.00 \mathrm{E}+00$ & $1.00 \mathrm{E}+01$ & $0.00 \mathrm{E}+00$ & $1.00 \mathrm{E}+00$ & $1.00 \mathrm{E}+04$ \\
\hline TANTALUM & & & $1.00 E+02$ & 1000 & $0.00 \mathrm{E}+00$ & $0.00 \mathrm{E}+00$ & 5.00E+01 & $3.98 \mathrm{E}-03$ & $0.00 \mathrm{E}+00$ \\
\hline TARTRATE (SODIUM) & & & 1.00E+02 & 1000 & $1.00 \mathrm{E}+00$ & $1.00 E+01$ & $0.00 \mathrm{E}+00$ & $1.00 \mathrm{E}+00$ & $1.00 \mathrm{E}+04$ \\
\hline TECHNETIUM(OXIDE) & & & $3.11 E+03$ & 1000 & $7.09 \mathrm{E}-01$ & $2.20 \mathrm{E}+02$ & $0.00 \mathrm{E}+00$ & $1.00 \mathrm{E}+00$ & $2.2 \overline{0 E+05}$ \\
\hline TELLURIUM & & & $1.00 \mathrm{E}+02$ & 1000 & $1.00 \mathrm{E}+00$ & $1.00 \mathrm{E}+01$ & $5.00 \mathrm{E}+01$ & $3.98 \mathrm{E}-03$ & $3.98 \mathrm{E}+01$ \\
\hline TETRABROMOETHANE & & & $1.00 E+02$ & 1000 & $1.00 \mathrm{E}+00$ & $1.00 E+01$ & $0.00 \mathrm{E}+00$ & $1.00 E+00$ & $1.00 \mathrm{E}+04$ \\
\hline TETRAHYDROFURAN & & & $1.00 E+02$ & 1000 & $1.00 \mathrm{E}+00$ & $1.00 \overline{\mathrm{E}+01}$ & $0.00 E+00$ & $1.00 \mathrm{E}+00$ & $1.00 \bar{E}+04$ \\
\hline TETRAPHENYL BORON & & & $1.00 \mathrm{E}+02$ & 1000 & $1.00 \mathrm{E}+00$ & $1.00 \mathrm{E}+01$ & $0.00 \mathrm{E}+00$ & $1.00 \mathrm{E}+00$ & $1.00 \mathrm{E}+04$ \\
\hline THALLIUM & & & $1.54 \bar{E}+02$ & 1000 & $1.00 \mathrm{E}+00$ & $1.54 \mathrm{E}+01$ & $5.00 \mathrm{E}+01$ & $3.98 E-03$ & $6.14 \mathrm{E}+01$ \\
\hline THENOYLTRIFLUOROACETONE & & & $1.00 \mathrm{E}+02$ & 1000 & $1.00 \mathrm{E}+00$ & $1.00 \mathrm{E}+01$ & $0.00 \mathrm{E}+00$ & $1.00 \mathrm{E}+00$ & $1.00 \mathrm{E}+04$ \\
\hline THIOCYANATE (AMMONIUM, Na) & & & $1.00 \mathrm{E}+02$ & 1000 & $1.00 \mathrm{E}+00$ & $1.00 \mathrm{E}+01$ & $0.00 E+00$ & $1.00 \mathrm{E}+00$ & $1.00 E+04$ \\
\hline THHOCYANATE (MERCURIC) & & & $1.00 E+02$ & 1000 & $1.00 \mathrm{E}+00$ & $1.00 \mathrm{E}+01$ & $0.00 \mathrm{E}+00$ & $1.00 \mathrm{E}+00$ & $1.00 \mathrm{E}+04$ \\
\hline THIOSULFATE (AMMONIUM, Na) & & & $1.00 \mathrm{E}+02$ & 1000 & $1.00 \mathrm{E}+00$ & $1.00 \mathrm{E}+01$ & $0.00 \mathrm{E}+00$ & $1.00 \mathrm{E}+00$ & $1.00 \mathrm{E}+04$ \\
\hline THORIUM & & & $2.79 \overline{\mathrm{E}+03}$ & 1000 & $0.00 \mathrm{E}+00$ & $0.00 \mathrm{E}+00$ & $1.00 \mathrm{E}+01$ & 1.96E-02 & $0.00 \mathrm{E}+00$ \\
\hline THYMOLPTHALEIN & & & $1.00 \mathrm{E}+02$ & 1000 & $1.00 \mathrm{E}+00$ & $1.00 \mathrm{E}+01$ & $0.00 \bar{E}+00$ & $1.00 \mathrm{E}+00$ & $1.00 \mathrm{E}+04$ \\
\hline TIN & & & $1.00 E+02$ & 1000 & $1.00 \mathrm{E}+00$ & $1.00 \mathrm{E}+01$ & $1.00 \mathrm{E}+01$ & 1.96E-02 & $1.96 \bar{E}+02$ \\
\hline TITANIUM & & & $1.55 E+02$ & 1000 & $0.00 \mathrm{E}+00$ & $0.00 E+00$ & $5.00 E+01$ & $3.98 \mathrm{E}-03$ & $0.00 \bar{E}+00$ \\
\hline TOC & $9.08 E+05$ & $8.97 E+05$ & $1.81 E+06$ & 1000 & $9.24 E-01$ & $1.67 \mathrm{E}+05$ & $0.00 \mathrm{E}+00$ & $1.00 \mathrm{E}+00$ & 1.67E+08 \\
\hline TOLUENE & & & $1.00 \mathrm{E}+02$ & 1 & $1.00 \mathrm{E}+00$ & $1.00 \mathrm{E}+01$ & $0.00 E+00$ & $1.00 \mathrm{E}+00$ & $1.00 \mathrm{E}+07$ \\
\hline TRICHLOROETHANE(12) & & & $0.00 \mathrm{E}+00$ & 0.2 & $1.00 E+00$ & $0 . \overline{00 E+00}$ & $0.00 \mathrm{E}+00$ & $1.00 \mathrm{E}+00$ & $0.00 \mathrm{E}+00$ \\
\hline TRIISOOCTYLAMINE & & & $1.00 E+02$ & 1000 & $1.00 \mathrm{E}+00$ & $1.00 \mathrm{E}+01$ & $0.00 E+00$ & $1.00 \mathrm{E}+00$ & $1.00 \mathrm{E}+04$ \\
\hline TRINDODECYLAMINE & & & $1.00 \mathrm{E}+02$ & 1000 & $1.00 \mathrm{E}+00$ & $1.00 \mathrm{E}+01$ & $0.00 \mathrm{E}+00$ & $1.00 \mathrm{E}+00$ & $1.00 \mathrm{E}+04$ \\
\hline TRINOCTYLAMINE & & & $1.00 \mathrm{E}+02$ & 1000 & $1.00 \mathrm{E}+00$ & $1.00 E+01$ & $0.00 \mathrm{E}+00$ & 1.00E+00 & $1.00 E+04$ \\
\hline TRIS(HYDRO.)AMINOMETHANE & & & $1.00 E+02$ & 1000 & $1.00 \bar{E}+00$ & $1.00 E+01$ & $0.00 E+00$ & $1.00 E+00$ & $1.00 E+04$ \\
\hline
\end{tabular}


WHC-SD-WM-TI-769

CHEMICAL CONSTITUENTS

SINGLE-SHEUL AND DOUBLE-SHELL TANKS

\begin{tabular}{|c|c|c|c|c|c|c|c|c|c|}
\hline & & & & WATER & & TANK & & & \\
\hline & SST & DST & TOTAL & QUALITY & ASSUMED & SOLUBLE & & & WATER \\
\hline TANK WASTE & MASS & MASS & MASS & STANDARD & SOLUBLE & RESIDUALS & ASSUMED & MOBILITY & HAZARD \\
\hline CONSTITUENT(1) & $(\mathrm{kg})(2)$ & $(\mathrm{kg})(2)$ & $(\mathbf{k g})(3)$ & $(\mathrm{mg} / \mathrm{L})(4)$ & FRACTION(5) & $(\mathrm{kg})(5)$ & $K d(6)$ & FACTOR(6) & INDEX(7) \\
\hline TUNGSTEN & & & $1.00 E+02$ & 1000 & $0.00 \mathrm{E}+00$ & $0.00 E+00$ & $0.00 \mathrm{E}+00$ & 1.00E+00 & $0.00 \mathrm{E}+00$ \\
\hline URANIUM & & & $1.60 \mathrm{E}+06$ & 1000 & $6.44 \mathrm{E}-01$ & $1.03 E+05$ & $0.00 \mathrm{E}+00$ & $1.00 \mathrm{E}+00$ & $1.03 E+08$ \\
\hline UREA & & & $1.00 \mathrm{E}+02$ & 1000 & $1.00 \mathrm{E}+00$ & $1.00 \mathrm{E}+\overline{0} 1$ & $0.00 \mathrm{E}+00$ & $1.00 \mathrm{E}+00$ & $1.00 E+04$ \\
\hline VANADIUM (PENTOXIDE) & & & $1.00 \mathrm{E}+02$ & 1000 & $0.00 \mathrm{E}+00$ & $0.00 E+00$ & $5.00 \mathrm{E}+01$ & $3.98 \mathrm{E}-03$ & $0.00 \bar{E}+00$ \\
\hline WATER & & & $5.08 \mathrm{E}+08$ & 1000 & $1.00 \mathrm{E}+00$ & $5.08 E+07$ & $0.00 \mathrm{E}+00$ & $1.00 \mathrm{E}+00$ & $5.08 E+10$ \\
\hline XYLENE & & & $1.00 \mathrm{E}+02$ & 10 & $1.00 \mathrm{E}+00$ & $1.00 \mathrm{E}+01$ & $0.00 \mathrm{E}+00$ & $1.00 \mathrm{E}+00$ & $1.00 E+06$ \\
\hline ZINC & & & $5.05 \mathrm{E}+02$ & 1000 & $0.00 \mathrm{E}+00$ & $0.00 E+00$ & $5.00 \mathrm{E}+01$ & $3.98 \mathrm{E}-03$ & $0.00 \mathrm{E}+00$ \\
\hline \multirow[t]{2}{*}{ ZIRCONIUM } & & & $1.21 \mathrm{E}+06$ & 1000 & $6.20 \mathrm{E}-03$ & $7.50 \mathrm{E}+02$ & $5.00 \mathrm{E}+01$ & $3,98 \mathrm{E}-03$ & $2.99 E+03$ \\
\hline & & & $7.59 \mathrm{E}+08$ & & & $7.3 \bar{E}+0 \overline{7}$ & & & \\
\hline
\end{tabular}

1
1
0 
WHC-SD-WM-TI-769

CHEMICAL CONSTITUENTS

SINGLE-SHELL AND DOUBLE-SHELL TANKS

\begin{tabular}{|c|c|c|c|c|c|}
\hline & & AlR & & & \\
\hline & MELTER & QUALITY & & AIRBORNE & \\
\hline TANK WASTE & FEED & STANDARD & VOLATILITY & HAZARD & \\
\hline CONSTITUENT(1) & $(\mathbf{k g})(5)$ & $(\mathrm{ug} / \mathrm{L})(8)$ & FACTOR(9) & INDEX(10) & TOXICITY(11) \\
\hline ACETATE & $8.96 \mathrm{E}+04$ & 1000000 & 1 & 8.96E-02 & \\
\hline ACETONE(12) & $0.00 \mathrm{E}+00$ & 2400 & 1 & $0.00 E+00$ & $D$ \\
\hline ALUMINUM & $3.86 \mathrm{E}+06$ & 1000000 & 0 & $0.00 E+00$ & \\
\hline AMMONIA (NH3) & $3.71 E+05$ & 35 & 1 & $1.06 \mathrm{E}+04$ & $B$ \\
\hline ANTIMONY & $9.00 \mathrm{E}+01$ & 0.5 & 0.25 & $4.50 \mathrm{E}+01$ & $\bar{x}$ \\
\hline ARSENIC & $1.17 \bar{E}+00$ & 0.5 & 1 & $2.33 E+00$ & $x$ \\
\hline BARIUM & $2.58 \mathrm{E}+03$ & 0.5 & 0.01 & $5.15 \mathrm{E}+01$ & C \\
\hline BENZENE(12) & $0.00 \mathrm{E}+00$ & 80 & 1 & $0.00 \mathrm{E}+00$ & C \\
\hline BERYLLIUM & $1.56 \mathrm{E}+00$ & 0.002 & 0.1 & $7.80 \mathrm{E}+01$ & $x$ \\
\hline BICARBONATE & $9.00 \mathrm{E}+01$ & 1000000 & 0.01 & 9.00E-07 & \\
\hline BISMUTH & $2.72 \mathrm{E}+05$ & 1000000 & 0.1 & $2.72 \mathrm{E}-02$ & \\
\hline BISULFATE (AMMONIUM) & $9.00 \mathrm{E}+01$ & 1000000 & 1 & $9.00 \mathrm{E}-05$ & \\
\hline BISULFITE & $9.00 E+01$ & 1000000 & 1 & $9.00 \mathrm{E}-05$ & \\
\hline BORON & $3.09 E+01$ & 1000000 & 0.25 & $7.72 \mathrm{E}-06$ & \\
\hline BROMATE & $9.00 \mathrm{E}+01$ & 1000000 & 1 & $9.00 \mathrm{E}-05$ & \\
\hline BROMINE & $9.00 \mathrm{E}+01$ & 0.7 & 1 & $1.29 E+02$ & \\
\hline BROMOCRESOL PURPLE & $9.00 \overline{\mathrm{E}}+01$ & 1000000 & 1 & $9.00 \mathrm{E}-05$ & \\
\hline BROMONAPHTHALENE & $9.00 E+01$ & 1000000 & 1 & $9.00 E-05$ & \\
\hline BUTANOL (BUTYL ALCOHOL) & $1.79 E+05$ & 1000000 & 1 & 1.79E-01 & D \\
\hline $\mathrm{C} 6 \mathrm{H} 507$ & $6.10 \mathrm{E}+05$ & 1000000 & 1 & 6.10E-01 & \\
\hline CADMIUM & $1.02 E+04$ & 0.2 & 0.5 & $2.54 \mathrm{E}+04$ & $\mathrm{X}$ \\
\hline CALCIUM & $3.35 \mathrm{E}+05$ & 1000000 & 0.01 & $3.35 \mathrm{E}-03$ & \\
\hline CANCRINITE & $1.35 \mathrm{E}+06$ & 1000000 & 0.01 & 1.35E-02 & \\
\hline CARBIDE (BORON) & $5.00 \mathrm{E}+01$ & 1000000 & 0.01 & $5.00 \mathrm{E}-07$ & \\
\hline CARBON TETRACHLORIDE(12) & $0.00 \mathrm{E}+00$ & 65 & 1 & $0.00 \mathrm{E}+00$ & D \\
\hline CARBONATE & $4.28 E+06$ & 1000000 & 0.01 & $4.28 \mathrm{E}-02$ & \\
\hline CERIUM & $1.19 \mathrm{E}+05$ & 1000000 & 0.01 & 1.19E-03 & \\
\hline CESIUM & $1.80 \mathrm{E}+03$ & 1000000 & 0.5 & $8.99 E-04$ & \\
\hline CHLORIDE & $8.56 \mathrm{E}+05$ & 1000000 & 1 & 8.56E-01 & \\
\hline CHROMIUM & $6.25 E+05$ & 1000000 & 0.1 & $6.25 \mathrm{E}-02$ & $\mathrm{X}$ \\
\hline COBALT & $2.12 \mathrm{E}+01$ & 0.1 & 0.25 & $5.29 \mathrm{E}+01$ & \\
\hline COPPER & $1.83 \mathrm{E}+02$ & 1 & 0.01 & $1.83 E+00$ & $x$ \\
\hline CYANIDE & $1.30 \mathrm{E}+05$ & 5 & 1 & $2.59 \mathrm{E}+04$ & $A$ \\
\hline
\end{tabular}


CHEMICAL CONSTITUENTS

SINGLE-SHELL AND DOUBLE-SHELL TANKS

\begin{tabular}{|c|c|c|c|c|c|}
\hline & & AIR & & & \\
\hline & MELTER & QUALITY & & AIRBORNE & \\
\hline TANK WASTE & FEED & STANDARD & VOLATILITY & HAZARD & \\
\hline CONSTITUENT(1) & $(\mathbf{k g})(5)$ & $(\mathbf{u g} / \mathrm{L})(8)$ & FACTOR(9) & INDEX(10) & TOXICITY(11) \\
\hline DIATOMACEOUS EARTH & $5.00 \mathrm{E}+04$ & 1000000 & 0.01 & 5.00E-04 & \\
\hline DIBUTYL PHOSPHANATE & $9.00 E+01$ & 1000000 & 1 & $9.00 \mathrm{E}-05$ & \\
\hline DIBUTYUTRIBUTYL PHOSPHATE & $5.35 E+05$ & 5 & 1 & 1.07E+05 & \\
\hline DICHROMATE & $5.00 \mathrm{E}+01$ & 1000000 & 0.1 & 5.00E-06 & \\
\hline DIHYDROXYANTHRAQUINONE(12) & $0.00 \mathrm{E}+00$ & 1000000 & 1 & $0.00 E+00$ & \\
\hline DIOXANE(12) & $0.00 \mathrm{E}+00$ & 360 & 1 & $0.00 \mathrm{E}+00$ & \\
\hline DTPA & $9.00 \mathrm{E}+01$ & 1000000 & 1 & $9.00 \mathrm{E}-05$ & \\
\hline DUODECANE & $9.00 \mathrm{E}+01$ & 1000000 & 1 & 9.00 E-05 & \\
\hline EDTA & $5.57 \bar{E}+05$ & 1000000 & 1 & 5.57E-01 & D \\
\hline ETHANOL & $9.00 E+01$ & 1900 & 1 & 4.74E-02 & \\
\hline ETHYL ETHER(12) & $0.00 \mathrm{E}+00$ & 1200 & 1 & $0.00 \mathrm{E}+00$ & \\
\hline ETHYLENE GLYCOL & $9.00 E+01$ & 1.2 & 1 & $7.50 E+01$ & \\
\hline FERROUS SULFAMATE & $9.00 E+02$ & 1000000 & 1 & $9.00 \mathrm{E}-04$ & \\
\hline FLUORIDE & $1.06 E+06$ & 1000000 & 1 & $1.06 \mathrm{E}+00$ & \\
\hline FLUOROSILICATE (AMMONIUM) & $9.00 E+01$ & 1000000 & 1 & $9.00 \mathrm{E}-05$ & \\
\hline FORMALDEHYDE(12) & $0.00 E+00$ & 6 & 1 & $0.00 \mathrm{E}+00$ & C \\
\hline GALLIUM & $9.00 \mathrm{E}+01$ & 1000000 & 0.01 & $9.00 \mathrm{E}-07$ & \\
\hline GLUCONATE (SODIUM) & $9.00 E+01$ & 1000000 & 1 & $9.00 \mathrm{E}-05$ & \\
\hline GLYCEROL & $9.00 \mathrm{E}+01$ & 1000000 & 1 & $9.00 \mathrm{E}-05$ & \\
\hline GLYCOLATE & $9.90 E+05$ & 1000000 & 1 & $9.90 \mathrm{E}-01$ & \\
\hline GOLD & $0.00 E+00$ & 1000000 & 0.01 & $0.00 \mathrm{E}+00$ & \\
\hline HEDTA & $9.31 E+05$ & 1000000 & 1 & 9.31E-01 & \\
\hline HYDRAZINE(12) & $0.00 \mathrm{E}+00$ & 1.3 & 1 & $0.00 \mathrm{E}+00$ & $\mathrm{X}$ \\
\hline HYDROGEN SULFIDE(12) & $0.00 \mathrm{E}+00$ & 15 & 1 & $0.00 \mathrm{E}+00$ & $B$ \\
\hline HYDROXIDE & $1.57 E+07$ & 2 & 0.01 & $7.83 E+04$ & \\
\hline HYDROXYLAMINE (NITRATE) & $9.00 E+01$ & 1000000 & 1 & $9.00 \mathrm{E}-05$ & \\
\hline HYOROXYLAMINE HYDR_(12) & $0.00 \mathrm{E}+00$ & 1000000 & 1 & $0.00 \mathrm{E}+00$ & \\
\hline HYDROXYQUINOLINE(12) & $0.00 E+00$ & 1000000 & 1 & $0.00 \mathrm{E}+00$ & \\
\hline HYPOCHLORITE (SODIUM) & $9.00 E+00$ & 1000000 & 1 & $9.00 \mathrm{E}-06$ & \\
\hline IODINE(12) & $0.00 E+00$ & 1 & 1 & $0,00 \mathrm{E}+00$ & \\
\hline IRON & $9.44 E+05$ & 1000000 & 0.01 & $9.44 \mathrm{E}-03$ & C \\
\hline ISOAMYL ACETATE & $9.00 \mathrm{E}+01$ & 525 & 1 & 1.71E-01 & \\
\hline ISOPROPYL ALCOHOL(12) & $0.00 \mathrm{E}+00$ & 900 & 1 & $0.00 \mathrm{E}+00$ & \\
\hline
\end{tabular}


WHC-SD-WM-TI-769

CHEMICAL CONSTITUENTS

SINGLE-SHELL AND DOUBLE-SHELL TANKS

\begin{tabular}{|c|c|c|c|c|c|}
\hline & & AIR & & & \\
\hline & MELTER & QUALITY & & AIRBORNE & \\
\hline TANK WASTE & FEED & STANDARD & VOLATILITY & HAZARD & \\
\hline CONSTITUENT(1) & $(\mathbf{k g})(5)$ & (ug/L)(8) & FACTOR(9) & INDEX(10) & TOXICITY(11) \\
\hline KEROSENE & $9.00 \mathrm{E}+01$ & 1000000 & 1 & 9.00E-05 & \\
\hline LANTHANUM & $2.04 E+04$ & 1000000 & 0.01 & $2.04 \mathrm{E}-04$ & \\
\hline LEAD & 1.67E+05 & 0.2 & 0.5 & $4.17 \mathrm{E}+05$ & $x$ \\
\hline LITHIUM & $9.00 \mathrm{E}+01$ & 1000000 & $\overline{0.1}$ & 9.00 E-06 & \\
\hline MAGNESIUM & $4.13 \mathrm{E}+03$ & 15 & 0.01 & $2.75 E+00$ & \\
\hline MANGANESE & $9.93 E+04$ & 1000000 & 0.01 & 9.93E-04 & \\
\hline MERCURY & $4.52 \mathrm{E}+03$ & 0.01 & 1 & $4.52 \mathrm{E}+05$ & $\mathrm{X}$ \\
\hline METHANOL(12) & $0.00 \mathrm{E}+00$ & 260 & 1 & $0.00 E+00$ & D \\
\hline METHYL ETHYL KEYTONE(12) & $0.00 \mathrm{E}+00$ & 1000000 & 1 & $0.00 E+00$ & D \\
\hline METHYL ISOBUTYL KETONE(12) & $0.00 \mathrm{E}+00$ & 1000000 & 1 & $0.00 E+00$ & $D$ \\
\hline MOLYBDENUM & $2.26 \mathrm{E}+01$ & 5 & 0.5 & $2.26 E+00$ & \\
\hline MORPHOLINE & $9.00 E+01$ & 70 & 1 & $1.29 \mathrm{E}+00$ & \\
\hline NAPHTHYLAMINE & $9.00 E+00$ & 0.002 & 1 & $4.50 \mathrm{E}+03$ & $x$ \\
\hline NEODYMIUM & $5.00 E+01$ & 1000000 & 0.01 & 5.00E-07 & \\
\hline NICKEL & $1.10 \mathrm{E}+05$ & 1 & 0.1 & $1.10 \mathrm{E}+04$ & $\mathrm{X}$ \\
\hline NIOBIUM & $9.00 \mathrm{E}+01$ & 1000000 & 0.01 & 9.00E-07 & \\
\hline NITRATE & $9.68 E+07$ & 1000000 & 1 & $9.68 \mathrm{E}+01$ & \\
\hline NITRITE & 1.22E+07 & 1000000 & 1 & $1.22 \mathrm{E}+01$ & \\
\hline OPHENATROLINE & $9.00 \mathrm{E}+01$ & 1000000 & 1 & 9.00E-05 & \\
\hline OXALATE (SODIUM) & $6.23 E+04$ & 1000000 & 1 & 6.23E-02 & \\
\hline PARAFFIN (NORMAL) HYDROCAR. & $5.00 E+01$ & 1000000 & 1 & $5.00 \mathrm{E}-05$ & \\
\hline PERMANGANATE (POTASSIUM) & $9.00 \mathrm{E}+01$ & 1000000 & 1 & $9.00 E-05$ & B \\
\hline PERSULFATE (POTASSIUM) & $9.00 E+01$ & 1000000 & 1. & $9.00 \mathrm{E}-05$ & \\
\hline PERSULFATE (SODIUM) & $9.00 \mathrm{E}+01$ & 1000000 & 1 & 9.00E-05 & \\
\hline PHOSPHATE & $3.03 E+06$ & 1000000 & 0.25 & 7.58E-01 & \\
\hline PHOSPHORUS PENTOXIDE & $9.00 \mathrm{E}+01$ & 1000000 & 0.25 & $2.25 \mathrm{E}-05$ & \\
\hline PLATINUM & $0.00 \mathrm{E}+00$ & 0.002 & 0.01 & $0.00 E+00$ & \\
\hline POTASSIUM & $6.51 \mathrm{E}+05$ & 1000000 & 0.25 & 1.63E-01 & \\
\hline PRASEODYMIUM & $5.00 \mathrm{E}+01$ & 1000000 & 0.01 & 5.00E-07 & \\
\hline RHENIUM & 4.87E-02 & 1000000 & 0.01 & 4.87E-10 & \\
\hline RHODIUM & $1.75 \mathrm{E}+02$ & 1000000 & 0.01 & 1.75E-06 & \\
\hline RUBIDIUM & $5.85 \mathrm{E}+00$ & 1000000 & 0.01 & 5.85E-08 & \\
\hline RUTHENIUM & $1.05 E+00$ & 1000000 & 1 & 1.05E-06 & \\
\hline
\end{tabular}


WHC-SD-WM-TI-769

CHEMICAL CONSTITUENTS

SINGLE-SHELL AND DOUBLE-SHELL TANKS

\begin{tabular}{|c|c|c|c|c|c|}
\hline & & AIR & & & \\
\hline & MELTER & QUALITY & & AIRBORNE & \\
\hline TANK WASTE & FEED & STANDARD & VOLATILITY & HAZARD & \\
\hline CONSTITUENT(1) & $(\mathbf{k g})(5)$ & (ug/L)(8) & FACTOR(9) & INDEX(10) & TOXICITY(11) \\
\hline SDIPHENYL CARBAZIDE & $5.00 \mathrm{E}+01$ & 1000000 & 0.01 & $5.00 \mathrm{E}-07$ & \\
\hline SELENIUM & $1.66 \mathrm{E}+03$ & 0.2 & 1 & $8.28 \mathrm{E}+03$ & $x$ \\
\hline SILICON (S|LICATE) & $3.22 \mathrm{E}+05$ & 1000000 & 0.01 & $3.22 \mathrm{E}-03$ & \\
\hline SILVER & $1.03 E+03$ & 0.01 & 0.25 & $2.57 \mathrm{E}+04$ & C \\
\hline SODIUM & $6.16 \mathrm{E}+07$ & 1000000 & 0.25 & $1.54 \mathrm{E}+01$ & A \\
\hline STRONTIUM & $1.86 E+04$ & 1000000 & 0.01 & 1.86E-04 & \\
\hline STYRENE & $9.00 \mathrm{E}+00$ & 420 & 1 & 2.14E-02 & C \\
\hline SUGAR & $9.00 E+04$ & 1000000 & 1 & $9.00 \mathrm{E}-02$ & \\
\hline SULFATE & $2.88 \mathrm{E}+06$ & 1000000 & 1 & $2.88 \mathrm{E}+00$ & \\
\hline SULFITE (AM MONIUM) & $9.00 \mathrm{E}+01$ & 1000000 & 1 & $9.00 \mathrm{E}-05$ & D \\
\hline TANTALUM & $5.00 \mathrm{E}+01$ & 5 & 0.01 & $1.00 \mathrm{E}-01$ & \\
\hline TARTRATE (SODIUM) & $9.00 \mathrm{E}+01$ & 1000000 & 1 & $9.00 \mathrm{E}-05$ & \\
\hline TECHNETIUM(OXIDE) & $2.44 E+03$ & 1000000 & 1 & 2.44E-03 & \\
\hline TELLURIUM & $9.00 \mathrm{E}+01$ & 0.1 & 1 & $9.00 \mathrm{E}+02$ & \\
\hline TETRABROMOETHANE & $9.00 \mathrm{E}+01$ & 1000000 & 1 & 9.00E-05 & \\
\hline TETRAHYDROFURAN & $9.00 \mathrm{E}+01$ & 590 & 1 & 1.53E-01 & \\
\hline TETRAPHENYL BORON & $9.00 \mathrm{E}+01$ & 1000000 & 1 & $9.00 \mathrm{E}-05$ & \\
\hline THALLIUM & $1.39 \mathrm{E}+02$ & 0.1 & 1 & $1.39 \mathrm{E}+03$ & $x$ \\
\hline THENOYLTRIFLUOROACETONE & $9.00 \mathrm{E}+01$ & 1000000 & 1 & $9.00 \mathrm{E}-05$ & \\
\hline THIOCYANATE (AMMONIUM, Na) & $9.00 \mathrm{E}+01$ & 1000000 & 1 & $9.00 \mathrm{E}-05$ & \\
\hline THIOCYANATE (MERCURIC) & $9.00 \mathrm{E}+01$ & 1000000 & 1 & 9.00E-05 & \\
\hline THIOSULFATE (AMMONIUM, Na) & $9.00 \mathrm{E}+01$ & 1000000 & 1 & 9.00E-05 & \\
\hline THORIUM & $1.40 \mathrm{E}+03$ & 1000000 & 0.01 & $1.40 \mathrm{E}-05$ & \\
\hline THYMOLPTHALEIN & $9.00 \mathrm{E}+01$ & 1000000 & 1 & $9.00 \mathrm{E}-05$ & \\
\hline TIN & $9.00 \mathrm{E}+01$ & 2 & 0.25 & $1.13 E+01$ & \\
\hline TITANIUM & $7.75 \mathrm{E}+01$ & 15 & 0.01 & 5.17E-02 & \\
\hline TOC & $1.57 \mathrm{E}+06$ & 1000000 & 1 & 1.57E+00 & $x$ \\
\hline TOLUENE & $9.00 \mathrm{E}+01$ & 750 & 1 & $1.20 \mathrm{E}-01$ & C \\
\hline TRICHLOROETHANE(12) & $0.00 \mathrm{E}+00$ & 1000000 & 1 & $0.00 E+00$ & $x$ \\
\hline TRIISOOCTYLAMINE & $9.00 \mathrm{E}+01$ & 1000000 & 1 & $9.00 \mathrm{E}-05$ & \\
\hline TRINDODECYLAMINE & $9.00 E+01$ & 1000000 & 1 & $9.00 E-05$ & \\
\hline TRINOCTYLAMINE & $9.00 \mathrm{E}+01$ & 1000000 & 1 & $9.00 \mathrm{E}-05$ & \\
\hline TRIS(HYDRO.)AMINOMETHANE & $9.00 \mathrm{E}+01$ & 1000000 & 1 & $9.00 \mathrm{E}-05$ & \\
\hline
\end{tabular}


WHC-SD-WM-TI-769

CHEMICAL CONSTITUENTS

SINGLE-SHELL AND DOUBLE-SHELL TANKS

\begin{tabular}{|c|c|c|c|c|c|}
\hline & & AIR & & & \\
\hline & MELTER & QUALITY & & AIRBORNE & \\
\hline TANK WASTE & FEED & STANDARD & VOLATILITY & HAZARD & \\
\hline CONSTITUENT(1) & $(\mathrm{kg})(5)$ & (ug/L)(8) & FACTOR(9) & INDEX(10) & TOXICITY(11) \\
\hline TUNGSTEN & $5.00 \mathrm{E}+01$ & 5 & 0.01 & $1.00 \mathrm{E}-01$ & \\
\hline URANIUM & $1.21 \mathrm{E}+06$ & 0.05 & 0.01 & $2.42 \mathrm{E}+05$ & \\
\hline UREA & $9.00 \mathrm{E}+01$ & 1000000 & 1 & $9.00 \mathrm{E}-05$ & \\
\hline VANADIUM (PENTOXIDE) & $5.00 \mathrm{E}+01$ & 0.5 & 0.01 & $1.00 \mathrm{E}+00$ & $\mathrm{C}$ \\
\hline WATER & 4.57E+08 & 1000000 & 1 & 4.57E+02 & \\
\hline XYLENE & $9.00 E+01$ & 435 & 1 & 2.07E-01 & C \\
\hline ZINC & $2.53 E+02$ & 1000000 & 0.5 & $1.26 \mathrm{E}-04$ & $x$ \\
\hline ZIRCONIUM & $6.08 \mathrm{E}+05$ & 5 & 0.01 & $1.22 E+03$ & \\
\hline
\end{tabular}


1. These are the constituents that are potentially in Hanford Site waste tanks, according to either Agnew (Agnew 1996), Shelton (Shelton 1996) or Klem (Klem 1990). The TOC includes all organic. Combinations of the constituents are not listed. The first word of the constituent designation is the anion or cation that is being compared. Constituents in bold (or their major tank compounds) are toxic (see note 11).

2. SST and DST masses are taken from LA-UR-96-858 (Agnew 1996).

3. Masses are from Agnew (Agnew 1996) or Shelton (Shelton 1996), except that values in bold are bounding estimates based on historical procurement records (e.g., Olson 1992). If masses are listed by both Shelton and Agnew, Agnew's numbers were used.

4. The numbers are groundwater quality standards (nonradiological only) from the Environmental Protection Manual (WHC 1993) or the maximum contaminant levels (MCL) of the federal drinking water standard, also taken from the Environmental Protection Manual. If no MCL or comparable standard exists, a value of $1000 \mathrm{mg} / \mathrm{L}$ was assigned for calculation purposes.

5. The assumed soluble fractions are derived from Shelton 1996. The soluble tank residuals are the estimated maximum amounts of soluble constituents remaining in the tanks after saltwell pumping and sludge washing. The soluble tank residuals are the Assumed Soluble Fractions (column 6) times the Total Mass (column 4) times $10 \%$ assumed residual. It is assumed that a minimum of $50 \%$ of insoluble material is retrieved. The melter feed, then, consists of $90 \%$ of the soluble plus $50 \%$ of the insoluble material.

6. The Kd values were taken from the TWRS EIS (DOE 1996). The mobility factor considers the mobility of the chemical in migrating to the groundwater. The mobility factor (MF) is given by: $M F=1 /(1+(5 \times \mathrm{Kd}))$, where the $\mathrm{Kd}$ is the distribution coeficient taken from DOE 1996.

7. This column is the quotient of the soluble inventory $(\mathrm{g})$ and the groundwater quality standard $(g / L)$, times the mobility factor. Consequently, the index is in units of liters.

8. The air quality standards (nonradiological only) are the Threshold Limiting Values (TLV) given by the American Conference of Governmental Industrial hygienists (ACGIH 1967). If no TLV exists, a value of $1 \mathrm{~g} / \mathrm{L}$ was assigned for calculation purposes.

9. The volatility factor is the estimated fraction of a constituent that would go airborne under melter conditions (Stegen 1994). All organic constituents are given a factor of 1, all refractories are arbitrarily given a value of .01 . Constituents such as nitrate and nitrite that evolve into gases or vapors under melter conditions were given a value of 1 .

10. This column is the quotient of the soluble and insoluble inventory (g) and the air quality standard $(g / L) \times$ the volatility factor.

11. The 10 CFR 302 , Table 302.4 toxicity category is given.

12. It is assumed that this chemical has been lost from the tanks either through evaporation or vaporization in the evaporators, because its boiling point is less than 100 degrees $\mathrm{C}$. 
WHC-SD-WM-TI-769, Rev. 0

APPENDIX B: CONSTITUENT PREVALENCE RANK 
WHC-SD-WM-TI-769

CONSTITUENT PREVALENCE RANK

\begin{tabular}{|c|c|c|c|c|c|c|c|}
\hline & & SOLUBLE & & & & & \\
\hline TANK WASTE & & \begin{tabular}{|l|} 
RESIDUALS \\
\end{tabular} & $\%$ & TANK WASTE & & FEED & $\%$ \\
\hline CONSTITUENT(1) & TOXICITY & $(\mathrm{kg})(2)$ & CUMULATIVE & CONSTITUENT(1) & TOXICITY & $(\mathbf{k g})(2)$ & CUMULATIVE \\
\hline WATER & & $5.08 \mathrm{E}+07$ & $69.34076 \%$ & WATER & & 4.57E+08 & $67.98836 \%$ \\
\hline NITRATE & & $1.07 \mathrm{E}+07$ & $83.94982 \%$ & NITRATE & & $9.68 \mathrm{E}+07$ & $82.38476 \%$ \\
\hline SODIUM & A & $6.80 E+06$ & $93.22816 \%$ & SODIUM & A & $6.16 \mathrm{E}+07$ & $91.54352 \%$ \\
\hline HYDROXIDE & & $1.43 E+06$ & $95.17846 \%$ & HYDROXIDE & & $1.57 \mathrm{E}+07$ & $93.87304 \%$ \\
\hline NITRITE & & $1.35 E+06$ & $97.02011 \%$ & NITRITE & & $1.22 E+07$ & $95.68382 \%$ \\
\hline PHOSPHATE & & $2.55 E+05$ & $98.43856 \%$ & PHOSPHATE & & $3.03 E+06$ & $97.34530 \%$ \\
\hline TOC & $x$ & $1.67 \mathrm{E}+05$ & $98.66621 \%$ & SULFATE & & $2.88 \mathrm{E}+06$ & $97.77376 \%$ \\
\hline FLUORIDE & & $1.14 \mathrm{E}+05$ & $98.82196 \%$ & TOC & $\mathrm{x}$ & 1.57E+06 & $98.00718 \%$ \\
\hline GLYCOLATE & & $1.10 \mathrm{E}+05$ & $98.97211 \%$ & CANCRINITE & & $1.35 \mathrm{E}+06$ & $98.20793 \%$ \\
\hline HEDTA & & $1.03 E+05$ & $99.11325 \%$ & URANIUM & & $1.21 \mathrm{E}+06$ & $98.38818 \%$ \\
\hline URANIUM & & $1.03 \mathrm{E}+05$ & $99.25389 \%$ & FLUORIDE & & $1.06 E+06$ & $98.54602 \%$ \\
\hline CHLORIDE & & $9.45 \mathrm{E}+04$ & $99.38282 \%$ & GLYCOLATE & & $9.90 \mathrm{E}+05$ & $98.69324 \%$ \\
\hline POTASSIUM & & $7.07 E+04$ & $99.47927 \%$ & IRON & C & $9.44 E+05$ & $98.83366 \%$ \\
\hline $\mathrm{C} 6 \mathrm{H} 5 \mathrm{O} 7$ & & $6.78 \mathrm{E}+04$ & $99.57181 \%$ & HEDTA & & $9.31 E+05$ & $98.97205 \%$ \\
\hline EDTA & D & $6.19 \mathrm{E}+04$ & $99.65630 \%$ & CHLORIDE & & $8.56 E+05$ & $99.09939 \%$ \\
\hline DIBUTYLTRIBUTYL PHOSPHATE & & $5.94 \mathrm{E}+04$ & $99.73738 \%$ & POTASSIUM & & $6.51 E+05$ & $99.19614 \%$ \\
\hline CHROMIUM & $x$ & $5.81 E+04$ & $99.81668 \%$ & CHROMIUM & $x$ & $6.25 \mathrm{E}+05$ & $99.28906 \%$ \\
\hline AMMONIA (NH3) & $\mathrm{B}$ & $4.13 E+04$ & $99.87299 \%$ & $\mathrm{C} 6 \mathrm{H} 5 \mathrm{O} 7$ & & $6.10 \mathrm{E}+05$ & $99.37980 \%$ \\
\hline BUTANOL (BUTYL ALCOHOL) & D & $1.98 \mathrm{E}+04$ & $99.90008 \%$ & ZIRCONIUM & & $6.08 E+05$ & $99.47021 \%$ \\
\hline CYANIDE & A & 1.44E+04 & $99.91973 \%$ & EDTA & D & $5.57 \mathrm{E}+05$ & $99.55306 \%$ \\
\hline SUGAR & & $1.00 E+04$ & $99.93338 \%$ & DIBUTYLTRIBUTYL PHOSPHATE & & $5.35 \mathrm{E}+05$ & $99.63256 \%$ \\
\hline ACETATE & & $9.95 E+03$ & $99.94696 \%$ & AMMONIA (NH3) & B & $3.71 \mathrm{E}+05$ & $99.68778 \%$ \\
\hline IRON & C & $7.32 E+03$ & $99.95695 \%$ & CALCIUM & & $3.35 \mathrm{E}+05$ & $99.73756 \%$ \\
\hline OXALATE (SODIUM) & & $6.92 E+03$ & $99.96640 \%$ & SHLICON (SILICATE) & & $3.22 E+05$ & $99.78547 \%$ \\
\hline LEAD & $x$ & $6.88 \mathrm{E}+03$ & $99.97579 \%$ & BISMUTH & & $2.72 E+05$ & $99.82585 \%$ \\
\hline CALCIUM & & $6.31 E+03$ & $99.98441 \%$ & BUTANOL (BUTYL ALCOHOL) & D & $1.79 \mathrm{E}+05$ & $99.85241 \%$ \\
\hline SILICON (SILICATE) & & $3.92 \mathrm{E}+03$ & $99.98977 \%$ & LEAD & $x$ & 1.67E+05 & $99.87722 \%$ \\
\hline BISMUTH & & $2.36 E+03$ & $99.99299 \%$ & CYANIDE & A & $1.30 \mathrm{E}+05$ & $99.89650 \%$ \\
\hline MANGANESE & & $1.08 \mathrm{E}+03$ & $99.99446 \%$ & CERIUM & & $1.19 \mathrm{E}+05$ & $99.91426 \%$ \\
\hline NICKEL & $x$ & $8.13 E+02$ & $99.99557 \%$ & NICKEL & $\mathrm{X}$ & $1.10 \mathrm{E}+05$ & $99.93065 \%$ \\
\hline CADMIUM & $x$ & $7.64 \mathrm{E}+02$ & $99.99662 \%$ & MANGANESE & & $9.93 E+04$ & $99.94543 \%$ \\
\hline
\end{tabular}




\begin{tabular}{|c|c|c|c|c|c|c|c|}
\hline & & SOLUBLE & & & & & \\
\hline & & TANK & & & & MELTER & \\
\hline TANK WASTE & & RESIDUALS & $\%$ & TANK WASTE & & FEED & $\%$ \\
\hline CONSTITUENT(1) & TOXICITY & $(\mathrm{kg})(2)$ & CUMULATIE & CONSTITUENT(1) & TOXICITY & $(\mathrm{kg})(2)$ & CUMULATIVE \\
\hline ZIRCONIUM & & $7.50 \mathrm{E}+02$ & $99.99764 \%$ & SUGAR & & $9.00 \mathrm{E}+04$ & $99.95881 \%$ \\
\hline MERCURY & $\mathrm{X}$ & $2.73 E+02$ & $99.99801 \%$ & ACETATE & & $8.96 \mathrm{E}+04$ & $99.97213 \%$ \\
\hline CERIUM & & $2.37 \mathrm{E}+02$ & $99.99834 \%$ & OXALATE (SOOIUM) & & $6.23 E+04$ & $99.98138 \%$ \\
\hline TECHNETIUM(OXIDE) & & $2.20 \mathrm{E}+02$ & $99.99864 \%$ & DIATOMACEOUS EARTH & & $5.00 \mathrm{E}+04$ & $99.98882 \%$ \\
\hline CESIUM & & $1.88 E+02$ & $99.99890 \%$ & LANTHANUM & & $2.04 E+04$ & $99.99185 \%$ \\
\hline FERROUS SULFAMATE & & $1.00 \mathrm{E}+02$ & $99.99903 \%$ & STRONTIUM & & 1.86E+04 & $99.99462 \%$ \\
\hline BARIUM & C & $7.79 \mathrm{E}+01$ & $99.99914 \%$ & CADMIUM & $\mathrm{x}$ & $1.02 \mathrm{E}+04$ & $99.99613 \%$ \\
\hline LANTHANUM & & $4.04 \mathrm{E}+01$ & $99.99919 \%$ & MERCURY & $x$ & $4.52 \bar{E}+03$ & $99.99680 \%$ \\
\hline STRONTIUM & & $3.69 \mathrm{E}+01$ & $99.99924 \%$ & MAGNESIUM & & $4.13 E+03$ & $99.99741 \%$ \\
\hline SILVER & C & $3.20 \mathrm{E}+01$ & $99.99929 \%$ & BARIUM & C & $2.58 \mathrm{E}+03$ & $99.99779 \%$ \\
\hline RHODIUM & & $1.94 E+01$ & $99.99931 \%$ & TECHNETIUM(OXIDE) & & $2.44 E+03$ & $99.99816 \%$ \\
\hline THALLIUM & $\mathrm{X}$ & 1.54E+01 & $99.99934 \%$ & CESIUM & & $1.80 \mathrm{E}+03$ & $99.99842 \%$ \\
\hline ANTIMONY & $x$ & $1.00 \mathrm{E}+01$ & $99.99935 \%$ & SELENIUM & $x$ & $1.66 \mathrm{E}+03$ & $99.99867 \%$ \\
\hline BICARBONATE & & $1.00 E+01$ & $99.99936 \%$ & THORIUM & & $1.40 \mathrm{E}+03$ & $99.99888 \%$ \\
\hline BISULFATE (AMMONIUM) & & $1.00 \mathrm{E}+01$ & $99.99938 \%$ & SILVER & C & $1.03 E+03$ & $99.99903 \%$ \\
\hline BISULFITE & & $1.00 \mathrm{E}+01$ & $99.99939 \%$ & FERROUS SULFAMATE & & $9.00 \mathrm{E}+02$ & $99.99916 \%$ \\
\hline BROMATE & & $1.00 \mathrm{E}+01$ & $99.99940 \%$ & ZINC & $\mathrm{X}$ & $2.53 E+02$ & $99.99920 \%$ \\
\hline BROMINE & & $1.00 \mathrm{E}+01$ & $99.99942 \%$ & COPPER & $\mathrm{X}$ & $1.83 E+02$ & $99.99923 \%$ \\
\hline BROMOCRESOL PURPLE & & $1.00 \mathrm{E}+01$ & $99.99943 \%$ & RHODIUM & & $1.75 \mathrm{E}+02$ & $99.99926 \%$ \\
\hline BROMONAPHTHALENE & & $1.00 \mathrm{E}+01$ & $99.99944 \%$ & THALLIUM & $x$ & $1.39 \mathrm{E}+02$ & $99.99928 \%$ \\
\hline DIBUTYL PHOSPHANATE & & $1.00 \mathrm{E}+01$ & $99.99946 \%$ & ANTIMONY & $x$ & $9.00 E+01$ & $99.99929 \%$ \\
\hline DTPA & & $1.00 \mathrm{E}+01$ & $99.99947 \%$ & BICARBONATE & & $9.00 E+01$ & $99.99930 \%$ \\
\hline DUODECANE & & $1.00 \mathrm{E}+01$ & $99.99949 \%$ & BISULFATE (AMMONIUM) & & $9.00 \mathrm{E}+01$ & $99.99932 \%$ \\
\hline ETHANOL & & $1.00 \mathrm{E}+01$ & $99.99950 \%$ & BISULFITE & & $9.00 \mathrm{E}+01$ & $99.99933 \%$ \\
\hline ETHYLENE GLYCOL & & $1.00 \mathrm{E}+01$ & $99.99951 \%$ & BROMATE & & $9.00 \mathrm{E}+01$ & $99.99934 \%$ \\
\hline FLUOROSILICATE (AMMONIUM) & & $1.00 E+01$ & $99.99953 \%$ & BROMINE & & $9.00 \mathrm{E}+01$ & $99.99936 \%$ \\
\hline GALLIUM & & $1.00 \mathrm{E}+01$ & $99.99954 \%$ & BROMOCRESOL PURPLE & & $9.00 E+01$ & $99.99937 \%$ \\
\hline GLUCONATE (SODIUM) & & $1.00 \mathrm{E}+01$ & $99.99955 \%$ & BROMONAPHTHALENE & & $9.00 \mathrm{E}+01$ & $99.99938 \%$ \\
\hline GLYCEROL & & $1.00 \mathrm{E}+01$ & $99.99957 \%$ & DIBUTYL PHOSPHANATE & & $9.00 E+01$ & $99.99940 \%$ \\
\hline HYDROXYLAMINE (NITRATE) & & $1.00 E+01$ & $99.99958 \%$ & DTPA & & $9.00 \mathrm{E}+01$ & $99.99941 \%$ \\
\hline ISOAMYL ACETATE & & $1.00 \mathrm{E}+01$ & $99.99959 \%$ & DUODECANE & & $9.00 \mathrm{E}+01$ & $99.99942 \%$ \\
\hline KEROSENE & & $1.00 \mathrm{E}+01$ & $99.99961 \%$ & ETHANOL & & $9.00 \mathrm{E}+01$ & $99.99944 \%$ \\
\hline LITHIUM & & $1.00 \mathrm{E}+01$ & $99.99962 \%$ & ETHYLENE GLYCOL & & $9.00 \mathrm{E}+01$ & $99.99945 \%$ \\
\hline
\end{tabular}


WHC-SD-WM-TI-769

CONSTITUENT PREVALENCE RANK

\begin{tabular}{|c|c|c|c|c|c|c|c|}
\hline & & SOLUBLE & & & & & \\
\hline & & TANK & & & & MELTER & \\
\hline TANK WASTE & & RESIDUALS & $\%$ & TANK WASTE & & FEED & $\%$ \\
\hline CONSTITUENT(1) & TOXICITY & $(\mathbf{k g})(2)$ & CUMULATIVE & CONSTITUENT(1) & TOXICITY & $(\mathrm{kg})(2)$ & CUMULATIVE \\
\hline MORPHOLINE & & $1.00 E+01$ & $99.99964 \%$ & FLUOROSILICATE (AMMONIUM) & & $9.00 E+01$ & $99.99946 \%$ \\
\hline NiOBIUM & & $1.00 E+01$ & $99.99965 \%$ & GALLIUM & & $9.00 \mathrm{E}+01$ & $99.99948 \%$ \\
\hline OPHENATROLINE & & $1.00 E+01$ & $99.99966 \%$ & GLUCONATE (SODIUM) & & $9.00 \mathrm{E}+01$ & $99.99949 \%$ \\
\hline PERMANGANATE (POTASSIUM) & $\bar{B}$ & $1.00 \mathrm{E}+01$ & $99.99968 \%$ & GLYCEROL & & $9.00 \mathrm{E}+01$ & $99.99950 \%$ \\
\hline PERSULFATE (POTASSIUM) & & $1.00 \mathrm{E}+01$ & $99.99969 \%$ & HYDROXYLAMINE (NITRATE) & & $9.00 \mathrm{E}+01$ & $99.99952 \%$ \\
\hline PERSULFATE (SODIUM) & & $1.00 \mathrm{E}+01$ & $99.99970 \%$ & ISOAMYL ACETATE & & $9.00 \mathrm{E}+01$ & $99.99953 \%$ \\
\hline PHOSPHORUS PENTOXIDE & & $1.00 \mathrm{E}+01$ & $99.99972 \%$ & KEROSENE & & $9.00 \mathrm{E}+01$ & $99.99954 \%$ \\
\hline SULFITE (AMMONIUM) & D & $1.00 \mathrm{E}+01$ & $99.99973 \%$ & LITHIUM & & $9.00 \mathrm{E}+01$ & $99.99956 \%$ \\
\hline TARTRATE (SODIUM) & & $1.00 \mathrm{E}+01$ & $99.99974 \%$ & MORPHOLINE & & $9.00 \mathrm{E}+01$ & $99.99957 \%$ \\
\hline TELLURIUM & & $1.00 \mathrm{E}+01$ & $99.99976 \%$ & NIOBIUM & & $9.00 \mathrm{E}+01$ & $99.99958 \%$ \\
\hline TETRABROMOETHANE & & $1.00 \mathrm{E}+01$ & $99.99977 \%$ & OPHENATROLINE & & $9.00 \mathrm{E}+01$ & $99.99960 \%$ \\
\hline TETRAHYDROFURAN & & $1.00 \mathrm{E}+01$ & $99.99979 \%$ & PERMANGANATE (POTASSIUM) & $\bar{B}$ & $9.00 \mathrm{E}+01$ & $99.99961 \%$ \\
\hline TETRAPHENYL BORON & & $1.00 \mathrm{E}+01$ & $99.99980 \%$ & PERSULFATE (POTASSIUM) & & $9.00 \mathrm{E}+01$ & $99.99962 \%$ \\
\hline THENOYLTRIFLUOROACETONE & & $1.00 \mathrm{E}+01$ & $99.99981 \%$ & PERSULFATE (SODIUM) & & $9.00 \mathrm{E}+01$ & $99.99964 \%$ \\
\hline THIOCYANATE (AMMONIUM, Na) & & $1.00 \mathrm{E}+01$ & $99.99983 \%$ & PHOSPHORUS PENTOXIDE & & $9.00 \mathrm{E}+01$ & $99.99965 \%$ \\
\hline THOCYANATE (MERCURIC) & & $1.00 \mathrm{E}+01$ & $99.99984 \%$ & SULFITE (AMMONIUM) & D & $9.00 \mathrm{E}+01$ & $99.99966 \%$ \\
\hline THIOSULFATE (AMMONIUM, Na) & & $1.00 \mathrm{E}+01$ & $99.99985 \%$ & TARTRATE (SODIUM) & & $9.00 \mathrm{E}+01$ & $99.99968 \%$ \\
\hline THYMOLPTHALEIN & & $1.00 \mathrm{E}+01$ & $99.99987 \%$ & TELLLURIUM & & $9.00 \mathrm{E}+01$ & $99.99969 \%$ \\
\hline TIN & & $1.00 \mathrm{E}+01$ & $99.99988 \%$ & TETRABROMOETHANE & & $9.00 \mathrm{E}+01$ & $99.99970 \%$ \\
\hline TOLUENE & C & $1.00 \mathrm{E}+01$ & $99.99989 \%$ & TETRAHYDROFURAN & & $9.00 \mathrm{E}+01$ & $99.99972 \%$ \\
\hline TRIISOOCTYLAMINE & & $1.00 \mathrm{E}+01$ & $99.99991 \%$ & TETRAPHENYL BORON & & $9.00 \mathrm{E}+01$ & $99.99973 \%$ \\
\hline TRINDODECYLAMINE & & 1.00E+01 & $99.99992 \%$ & THENOYLTRIFLUOROACETONE & & $9.00 \mathrm{E}+01$ & $99.99974 \%$ \\
\hline TRINOCTYLAMINE & & 1.00E+01 & $99.99994 \%$ & THIOCYANATE (AMMONIUM, Na) & & $9.00 \mathrm{E}+01$ & $99.99976 \%$ \\
\hline TRIS(HYDRO,)AMINOMETHANE & & $1.00 \mathrm{E}+01$ & $99.99995 \%$ & THIOCYANATE (MERCURIC) & & $9.00 \mathrm{E}+01$ & $99.99977 \%$ \\
\hline UREA & & $1.00 \mathrm{E}+01$ & $99.99996 \%$ & THIOSULFATE (AMMONIUM, Na) & & $9.00 \mathrm{E}+01$ & $99.99978 \%$ \\
\hline XYLENE & C & $1.00 \mathrm{E}+01$ & $99.99998 \%$ & THYMOLPTHALEIN & & $9.00 E+01$ & $99.99980 \%$ \\
\hline COPPER & $x$ & $9.87 \mathrm{E}+00$ & $99.99999 \%$ & TIN & & $9.00 \mathrm{E}+01$ & $99.99981 \%$ \\
\hline BORON & & $3.43 \mathrm{E}+00$ & $99.99999 \%$ & TOLUENE & C & $9.00 \mathrm{E}+01$ & $99.99982 \%$ \\
\hline HYPOCHLORITE (SODIUM) & & $1.00 E+00$ & $100.00000 \%$ & TRIISOOCTYLAMINE & & $9.00 \mathrm{E}+01$ & $99.99984 \%$ \\
\hline NAPHTHYLAMINE & $\mathrm{x}$ & $1.00 E+00$ & $100.00000 \%$ & TRINDODECYLAMINE & & $9.00 \mathrm{E}+01$ & $99.99985 \%$ \\
\hline STYRENE & C & $1.00 \mathrm{E}+00$ & $100.00000 \%$ & TRINOCTYLAMINE & & $9.00 \mathrm{E}+01$ & $99.99986 \%$ \\
\hline MOLYBDENUM & & $7.21 E-01$ & $100.00000 \%$ & TRIS(HYDRO.)AMINOMETHANE & & $9.00 \mathrm{E}+01$ & $99.99988 \%$ \\
\hline RUTHENIUM & & 1.17E-01 & $100.00000 \%$ & UREA & & $9.00 \mathrm{E}+01$ & $99.99989 \%$ \\
\hline
\end{tabular}




\begin{tabular}{|c|c|c|c|c|c|c|c|}
\hline & & SOLUBLE & & & - & & \\
\hline & & TANK & & & & MELTER & \\
\hline TANK WASTE & & RESIDUALS & $\%$ & TANK WASTE & & FEED & $\%$ \\
\hline CONSTITUENT(1) & TOXICITY & $(\mathbf{k g})(\mathbf{2})$ & CUMULATIVE & CONSTITUENT(1) & TOXICITY & $(\mathrm{kg})(2)$ & CUMULATIVE \\
\hline ARSENIC & $\mathrm{X}$ & $4.14 E-03$ & $100.00000 \%$ & XYLENE & $\mathrm{C}$ & $9.00 E+01$ & $99.99991 \%$ \\
\hline BERYLLIUM & $\mathrm{X}$ & $2.48 E-03$ & $100.00000 \%$ & TITANIUM & & $7.75 E+01$ & $99.99992 \%$ \\
\hline ACETONE(12) & $\bar{D}$ & $0.00 \mathrm{E}+00$ & $100.00000 \%$ & CARBIDE (BORON) & & $5.00 \mathrm{E}+01$ & $99.99992 \%$ \\
\hline ALUMINUM & & $0.00 \mathrm{E}+00$ & $100.00000 \%$ & DICHROMATE & & $5.00 \mathrm{E}+01$ & $99.99993 \%$ \\
\hline BENZENE(12) & C & $0.00 \mathrm{E}+00$ & $100.00000 \%$ & NEODYMIUM & & 5.00E+01 & $99.99994 \%$ \\
\hline CANCRINITE & & $0.00 \mathrm{E}+00$ & $100.00000 \%$ & PARAFFIN (NORMAL) HYDROCAR. & & $5.00 \mathrm{E}+01$ & $99.99995 \%$ \\
\hline CARBIDE (BORON) & & $0.00 E+00$ & $100.00000 \%$ & PRASEODYMIUM & & $5.00 \mathrm{E}+01$ & $99.99995 \%$ \\
\hline CARBON TETRACHLORIDE(12) & D & $0.00 \mathrm{E}+00$ & $100.00000 \%$ & SDIPHENYL CARBAZIDE & & $5.00 \mathrm{E}+01$ & $99.99996 \%$ \\
\hline COBALT & & $0.00 \mathrm{E}+00$ & $100.00000 \%$ & TANTALUM & & $5.00 \mathrm{E}+01$ & $99.99997 \%$ \\
\hline DIATOMACEOUS EARTH & & $0.00 \mathrm{E}+00$ & $100.00000 \%$ & TUNGSTEN & & $5.00 \mathrm{E}+01$ & $99.99998 \%$ \\
\hline DICHROMATE & & $0.00 E+00$ & $100.00000 \%$ & VANADIUM (PENTOXIDE) & $\mathrm{C}$ & $5.00 \mathrm{E}+01$ & $99.99998 \%$ \\
\hline DIHYDROXYANTHRAQUINONE(12) & & $0.00 \mathrm{E}+00$ & $100.00000 \%$ & BORON & & $3.09 E+01$ & $99.99999 \%$ \\
\hline DIOXANE(12) & & $0.00 \mathrm{E}+00$ & $100.00000 \%$ & MOLYBDENUM & & $2.26 \mathrm{E}+01$ & $99.99999 \%$ \\
\hline ETHYL ETHER(12) & & $0.00 \mathrm{E}+00$ & $100.00000 \%$ & COBALT & & $2.12 \mathrm{E}+01$ & $99.99999 \%$ \\
\hline FORMALDEHYDE(12) & C & $0.00 E+00$ & $100.00000 \%$ & HYPOCHLORITE (SODIUM) & & $9.00 \mathrm{E}+00$ & $100.00000 \%$ \\
\hline GOLD & & $0.00 E+00$ & $100.00000 \%$ & NAPHTHYLAMINE & $x$ & $9.00 E+00$ & $100.00000 \%$ \\
\hline HYDRAZINE(12) & $\mathrm{X}$ & $0.00 \mathrm{E}+00$ & $100.00000 \%$ & STYRENE & $\mathrm{C}$ & $9.00 \mathrm{E}+00$ & $100.00000 \%$ \\
\hline HYDROGEN SULFIDE(12) & $\bar{B}$ & $0.00 \mathrm{E}+00$ & $100.00000 \%$ & RUBIDIUM & & $5.85 E+00$ & $100.00000 \%$ \\
\hline HYDROXYLAMINE HYDR.(12) & & $0.00 \mathrm{E}+00$ & $100.00000 \%$ & BERYLLIUM & $\mathrm{X}$ & $1.56 E+00$ & $100.00000 \%$ \\
\hline HYDROXYQUINOLINE(12) & & $0.00 E+00$ & $100.00000 \%$ & ARSENIC & $x$ & $1.17 E+00$ & $100.00000 \%$ \\
\hline IODINE(12) & & $0.00 \mathrm{E}+00$ & $100.00000 \%$ & RUTHENIUM & & $1.05 E+00$ & $100.00000 \%$ \\
\hline ISOPROPYL ALCOHOL(12) & & $0.00 \mathrm{E}+00$ & $100.00000 \%$ & RHENIUM & & 4.87E-02 & $100.00000 \%$ \\
\hline MAGNESIUM & & $0.00 \mathrm{E}+00$ & $100.00000 \%$ & ACETONE(12) & $D$ & $0.00 E+00$ & $100.00000 \%$ \\
\hline METHANOL(12) & D & $0.00 \mathrm{E}+00$ & $100.00000 \%$ & BENZENE(12) & C & $0.00 \mathrm{E}+00$ & $100.00000 \%$ \\
\hline METHYL ETHYL KEYTONE(12) & D & $0.00 \mathrm{E}+00$ & $100.00000 \%$ & CARBON TETRACHLORIDE(12) & D & $0.00 E+00$ & $100.00000 \%$ \\
\hline METHYL ISOBUTYL KETONE(12) & D & $0.00 \mathrm{E}+00$ & $100.00000 \%$ & DIHYDROXYANTHRAQUINONE(12) & & $0.00 \mathrm{E}+00$ & $100.00000 \%$ \\
\hline NEODYMIUM & & $0.00 \mathrm{E}+00$ & $100.00000 \%$ & DIOXANE(12) & & $0.00 \mathrm{E}+00$ & $100.00000 \%$ \\
\hline PARAFFIN (NORMAL) HYDROCAR. & & $0.00 \mathrm{E}+00$ & $100.00000 \%$ & ETHYL ETHER(12) & & $0.00 \mathrm{E}+00$ & $100.00000 \%$ \\
\hline PLATINUM & & $0.00 \mathrm{E}+00$ & $100.00000 \%$ & FORMALDEHYDE(12) & $C$ & $0.00 \mathrm{E}+00$ & $100.00000 \%$ \\
\hline PRASEODYMIUM & & $0.00 \mathrm{E}+00$ & $100.00000 \%$ & GOLD & & $0.00 \mathrm{E}+00$ & $100.00000 \%$ \\
\hline RHENIUM & & $0.00 \mathrm{E}+00$ & $100.00000 \%$ & HYDRAZINE(12) & $x$ & $0.00 E+00$ & $100.00000 \%$ \\
\hline RUBIDIUM & & $0.00 \mathrm{E}+00$ & $100.00000 \%$ & HYDROGEN SULFIDE(12) & $B$ & $0.00 E+00$ & $100.00000 \%$ \\
\hline SDIPHENYL CARBAZIDE & & $0.00 \mathrm{E}+00$ & $100.00000 \%$ & HYDROXYLAMINE HYDR.(12) & & $0.00 \mathrm{E}+00$ & $100.00000 \%$ \\
\hline
\end{tabular}


WHC-SD-WM-TI-769

CONSTITUENT PREVALENCE RANK

\begin{tabular}{|c|c|c|c|c|c|c|c|}
\hline & & SOLUBLE & & & & & \\
\hline & & TANK & & & & MELTER & \\
\hline TANK WASTE & & RESIDUALS & $\%$ & TANK WASTE & & FEED & $\%$ \\
\hline CONSTITUENT(1) & TOXICITY & $(\mathbf{k g})(2)$ & CUMULATIVE & CONSTITUENT(1) & TOXICITY & $(\mathbf{k g})(2)$ & CUMULATIVE \\
\hline SELENIUM & $\mathrm{x}$ & $0.00 \mathrm{E}+00$ & $100.00000 \%$ & HYDROXYQUINOLINE(12) & & $0.00 \mathrm{E}+00$ & $100.00000 \%$ \\
\hline TANTALUM & & $0.00 E+00$ & $100.00000 \%$ & IODINE(12) & & $0.00 E+00$ & $100.00000 \%$ \\
\hline THORIUM & & $0.00 E+00$ & $100.00000 \%$ & ISOPROPYL ALCOHOL(12) & & $0.00 E+00$ & $100.00000 \%$ \\
\hline TITANIUM & & $0.00 \mathrm{E}+00$ & $100.00000 \%$ & METHANOL(12) & $\overline{\mathrm{D}}$ & $0.00 E+00$ & $100.00000 \%$ \\
\hline TRICHLOROETHANE(12) & $x$ & $0.00 E+00$ & $100.00000 \%$ & METHYL ETHYL KEYTONE(12) & D & $0.00 E+00$ & $100.00000 \%$ \\
\hline TUNGSTEN & & $0.00 \mathrm{E}+00$ & $100.00000 \%$ & METHYL ISOBUTYL KETONE(12) & $\mathrm{D}$ & $0.00 \mathrm{E}+00$ & $100.00000 \%$ \\
\hline VANADIUM (PENTOXIDE) & C & $0.00 \mathrm{E}+00$ & $100.00000 \%$ & PLATINUM & & $0.00 \mathrm{E}+00$ & $100.00000 \%$ \\
\hline \multirow[t]{2}{*}{ ZINC } & $\bar{x}$ & $0.00 E+00$ & $100.00000 \%$ & TRICHLOROETHANE(12) & $\bar{x}$ & $0.00 \mathrm{E}+00$ & $100.00000 \%$ \\
\hline & & $7.33 E+07$ & & & & $6.72 \mathrm{E}+08$ & \\
\hline & & & & & & & \\
\hline & & & & & & & \\
\hline & & & & & & & \\
\hline & & & & & & & \\
\hline & & & & & & & \\
\hline & & & & & & & \\
\hline NOTES: & & & & & & & \\
\hline & & & & & & & \\
\hline (1) The constituents (or & compounds & in bold are tox & xic. & & & & \\
\hline & & & & & & & \\
\hline (2) Tank residuals are & IImum IIK & y, assuming 10 & $0 \%$ of the solt & e material and $50 \%$ of the & & & \\
\hline
\end{tabular}


WHC-SD-WM-TI-769, Rev. 0

APPENDIX C: HAZARDOUS RANK OF CONSTITUENTS 


\begin{tabular}{|c|c|c|c|c|c|}
\hline & & $\%$ & & & $\%$ \\
\hline & WATER & CUMULATIVE & & AIRBORNE & CUMULATIVE \\
\hline TANK WASTE & HAZARD & WATER & TANK WASTE & HAZARD & AIRBORNE \\
\hline CONSTITUENT(1) & INDEX & HAZARD & CONSTITUENT & INDEX & HAZARD \\
\hline NITRITE & $1.35 \mathrm{E}+12$ & $41.19 \%$ & MERCURY & 4.52E+05 & $32.01 \%$ \\
\hline NITRATE & 1.07E+12 & $73.87 \%$ & LEAD & $4.17 \mathrm{E}+05$ & $61.55 \%$ \\
\hline CHROMIUM & 5.81E+11 & $91.61 \%$ & URANIUM & $2.42 E+05$ & $78.71 \%$ \\
\hline MERCURY & $1.37 E+11$ & $95.78 \%$ & DIBUTYUTRRIBUTYL PHOSPHATE & $1.07 \mathrm{E}+05$ & $86.28 \%$ \\
\hline LEAD & $6.75 \mathrm{E}+10$ & $97.84 \%$ & HYDROXIDE & $7.83 E+04$ & $91.82 \%$ \\
\hline FLUUORIDE & $2.85 E+10$ & $98.71 \%$ & CYANIDE & $2.59 \mathrm{E}+04$ & $93.66 \%$ \\
\hline CADMIUM & $2.55 \mathrm{E}+10$ & $99.48 \%$ & SILVER & $2.57 \mathrm{E}+04$ & $95.48 \%$ \\
\hline SODIUM & $6.80 E+09$ & $99.69 \%$ & CADMIUM & $2.54 E+04$ & $97.27 \%$ \\
\hline IRON & $4.07 E+09$ & $99.82 \%$ & NICKEL & $1.10 \mathrm{E}+04$ & $98.05 \%$ \\
\hline ANTIMONY & $1.67 E+09$ & $99.87 \%$ & AMMONIA (NH3) & $1.06 \mathrm{E}+04$ & $98.81 \%$ \\
\hline HYDROXIDE & $1.43 \bar{E}+09$ & $99.91 \%$ & SELENIUM & $8.28 E+03$ & $99.39 \%$ \\
\hline SULFATE & 1.27E+09 & $99.95 \%$ & NAPHTHYLAMINE & $4.50 \mathrm{E}+03$ & $99.71 \%$ \\
\hline MANGANESE & $4.25 E+08$ & $99.96 \%$ & THALLIUM & $1.39 \mathrm{E}+03$ & $99.81 \%$ \\
\hline CHLORIDE & $3.78 \mathrm{E}+08$ & $99.97 \%$ & ZIRCONIUM & $1.22 \mathrm{E}+03$ & $99.89 \%$ \\
\hline TOC & $1.67 E+08$ & $99.98 \%$ & TELLUR!UM & $9.00 \mathrm{E}+02$ & $99.96 \%$ \\
\hline GLYCOLATE & 1.10E+08 & $99.98 \%$ & BROMINE & $1.29 E+02$ & $99.97 \%$ \\
\hline HEDTA & $1.03 E+08$ & $99.99 \%$ & NITRATE & $9.68 \mathrm{E}+01$ & $99.97 \%$ \\
\hline URANIUM & $1.03 E+08$ & $99.99 \%$ & BERYLLUM & $7.80 \mathrm{E}+01$ & $99.98 \%$ \\
\hline POTASSIUM & 7.07E+07 & $99.99 \%$ & ETHYLENE GLYCOL & $7.50 \mathrm{E}+01$ & $99.99 \%$ \\
\hline $\mathrm{C} 6 \mathrm{H} 5 \mathrm{O} 7$ & $6.78 \mathrm{E}+07$ & $99.99 \%$ & COBALT & 5.29E+01 & $99.99 \%$ \\
\hline EDTA & $6.19 \mathrm{E}+07$ & $99.99 \%$ & BARIUM & $5.15 E+01$ & $99.99 \%$ \\
\hline DIBUTYLUTRIBUTYL PHOSPHATE & $5.94 \mathrm{E}+07$ & $100.00 \%$ & ANTIMONY & $4.50 \hat{\mathrm{E}}+01$ & $100.00 \%$ \\
\hline AMMONIA (NH3) & $4.13 E+07$ & $100.00 \%$ & SODIUM & $1.54 \mathrm{E}+01$ & $100.00 \%$ \\
\hline BUTANOL (BUTYL ALCOHOL) & $1.98 \mathrm{E}+07$ & $100.00 \%$ & NITRITE & $1.22 \mathrm{E}+01$ & $100.00 \%$ \\
\hline CYANIDE & $1.44 \mathrm{E}+07$ & $100.00 \%$ & $\mathrm{TIN}$ & $1.13 E+01$ & $100.00 \%$ \\
\hline SUGAR & $1.00 \mathrm{E}+07$ & $100.00 \%$ & SULFATE & $2.88 E+00$ & $100.00 \%$ \\
\hline TOLUENE & $1.00 E+07$ & $100.00 \%$ & MAGNESIUM & $2.75 \mathrm{E}+00$ & $100.00 \%$ \\
\hline ACETATE & $9.95 E+06$ & $100.00 \%$ & ARSENIC & $2.33 E+00$ & $100.00 \%$ \\
\hline OXALATE (SODIUM) & $6.92 E+06$ & $100.00 \%$ & MOLYBDENUM & $2.26 \mathrm{E}+00$ & $100.00 \%$ \\
\hline PHOSPHATE & $5.00 \mathrm{E}+06$ & $100.00 \%$ & COPPER & $1.83 \mathrm{E}+00$ & $100.00 \%$ \\
\hline CARBONATE & $1.86 \mathrm{E}+06$ & $100.00 \%$ & TOC & 1.57E+00 & $100.00 \%$ \\
\hline CALCIUM & $1.05 \mathrm{E}+06$ & $100.00 \%$ & MORPHOLINE & $1.29 \mathrm{E}+00$ & $100.00 \%$ \\
\hline XYLENE & $1.00 \mathrm{E}+06$ & $100.00 \%$ & FLUORIDE & $1.06 \mathrm{E}+00$ & $100.00 \%$ \\
\hline
\end{tabular}




\begin{tabular}{|c|c|c|c|c|c|}
\hline & & $\%$ & & & $\%$ \\
\hline & WATER & CUMULATIVE & & AIRBORNE & CUMULATIVE \\
\hline TANK WASTE & HAZARD & WATER & TANK WASTE & HAZARD & AIRBORNE \\
\hline CONSTITUENT(1) & INDEX & HAZARD & CONSTITUENT & INDEX & HAZARD \\
\hline BARIUM & $7.64 \mathrm{E}+05$ & $100.00 \%$ & VANADIUM (PENTOXIDE) & $1.00 \mathrm{E}+00$ & $100.00 \%$ \\
\hline BISMUTH & $3.93 \mathrm{E}+05$ & $100.00 \%$ & GLYCOLATE & $9.90 \mathrm{E}-01$ & $100.00 \%$ \\
\hline TECHNETIUM(OXIDE) & $2.20 \mathrm{E}+05$ & $100.00 \%$ & HEDTA & 9.31E-01 & $100.00 \%$ \\
\hline NICKEL & $1.36 \mathrm{E}+05$ & $100.00 \%$ & CHLORIDE & $8.56 \bar{E}-01$ & $100.00 \%$ \\
\hline FERROUS SULFAMATE & $1.00 \mathrm{E}+05$ & $100.00 \%$ & PHOSPHATE & $7.58 \mathrm{E}-01$ & $100.00 \%$ \\
\hline RHODIUM & $1.94 E+04$ & $100.00 \%$ & $\mathrm{C} 6 \mathrm{H} 5 \mathrm{O} 7$ & $6.10 E-01$ & $100.00 \%$ \\
\hline SILICON (SILICATE) & $1.56 \mathrm{E}+04$ & $100.00 \%$ & EDTA & $5.57 \mathrm{E}-01$ & $100.00 \%$ \\
\hline BISULFATE (AMMONIUM) & $1.00 \mathrm{E}+04$ & $100.00 \%$ & XYLENE & 2.07E-01 & $100.00 \%$ \\
\hline BISULFITE & $1.00 \mathrm{E}+04$ & $100.00 \%$ & BUTANOL (BUTYL ALCOHOL) & 1.79E-01 & $100.00 \%$ \\
\hline BROMATE & $1.00 \mathrm{E}+04$ & $100.00 \%$ & ISOAMYL ACETATE & 1.71E-01 & $100.00 \%$ \\
\hline BROMINE & $1.00 E+04$ & $100.00 \%$ & POTASSIUM & 1.63E-01 & $100.00 \%$ \\
\hline BROMOCRESOL PURPLE & $1.00 \mathrm{E}+04$ & $100.00 \%$ & TETRAHYDROFURAN & 1.53E-01 & $100.00 \%$ \\
\hline BROMONAPHTHALENE & $1.00 \mathrm{E}+04$ & $100.00 \%$ & TOLUENE & 1.20E-01 & $100.00 \%$ \\
\hline DIBUTYL PHOSPHANATE & $1.00 \mathrm{E}+04$ & $100.00 \%$ & TANTALUM & 1.00E-01 & $100.00 \%$ \\
\hline DTPA & $1.00 \mathrm{E}+04$ & $100.00 \%$ & TUNGSTEN & 1.00E-01 & $100.00 \%$ \\
\hline DUODECANE & $1.00 \mathrm{E}+04$ & $100.00 \%$ & SUGAR & $9.00 \mathrm{E}-02$ & $100.00 \%$ \\
\hline ETHANOL & $1.00 \mathrm{E}+04$ & $100.00 \%$ & ACETATE & $8.96 \mathrm{E}-02$ & $100.00 \%$ \\
\hline ETHYLENE GLYCOL & $1.00 \mathrm{E}+04$ & $100.00 \%$ & CHROMIUM & $6.25 \mathrm{E}-02$ & $100.00 \%$ \\
\hline FLUOROSILICATE (AMMONIUM) & $1.00 \mathrm{E}+04$ & $100.00 \%$ & OXALATE (SODIUM) & 6.23E-02 & $100.00 \%$ \\
\hline GLUCONATE (SODIUM) & $1.00 \mathrm{E}+04$ & $100.00 \%$ & TITANIUM & 5.17E-02 & $100.00 \%$ \\
\hline GLYCEROL & $1.00 \mathrm{E}+04$ & $100.00 \%$ & ETHANOL & 4.74E-02 & $100.00 \%$ \\
\hline HYDROXYLAMINE (NITRATE) & $1.00 E+04$ & $100.00 \%$ & CARBONATE & 4.28E-02 & $100.00 \%$ \\
\hline ISOAMYL ACETATE & $1.00 \mathrm{E}+04$ & $100.00 \%$ & BISMUTH & 2.72E-02 & $100.00 \%$ \\
\hline KEROSENE & $1.00 \mathrm{E}+04$ & $100.00 \%$ & STYRENE & 2.14E-02 & $100.00 \%$ \\
\hline LITHIUM & $1.00 \mathrm{E}+04$ & $100.00 \%$ & CANCRINITE & 1.35E-02 & $100.00 \%$ \\
\hline MORPHOLINE & $1.00 \mathrm{E}+04$ & $100.00 \%$ & IRON & $9.44 \mathrm{E}-03$ & $100.00 \%$ \\
\hline OPHENATROLINE & $1.00 \mathrm{E}+04$ & $100.00 \%$ & CALCIUM & 3.35E-03 & $100.00 \%$ \\
\hline PERMANGANATE (POTASSIUM) & $1.00 \mathrm{E}+04$ & $100.00 \%$ & SILICON (SILICATE) & $3.22 \mathrm{E}-03$ & $100.00 \%$ \\
\hline PERSULFATE (POTASSIUM) & $1.00 \mathrm{E}+04$ & $100.00 \%$ & TECHNETIUM(OXIDE) & $2.44 \mathrm{E}-03$ & $100.00 \%$ \\
\hline PERSULFATE (SODIUM) & $1.00 \mathrm{E}+04$ & $100.00 \%$ & CERIUM & 1.19E-03 & $100.00 \%$ \\
\hline PHOSPHORUS PENTOXIDE & $1.00 \mathrm{E}+04$ & $100.00 \%$ & MANGANESE & 9.93E-04 & $100.00 \%$ \\
\hline SULFITE (AMMONIUM) & $1.00 \mathrm{E}+04$ & $100.00 \%$ & FERROUS SULFAMATE & $9.00 \mathrm{E}-04$ & $100.00 \%$ \\
\hline TARTRATE (SODIUM) & $1.00 E+04$ & $100.00 \%$ & CESIUM & 8.99E-04 & $100.00 \%$ \\
\hline
\end{tabular}




\begin{tabular}{|c|c|c|c|c|c|}
\hline & & $\%$ & & & $\%$ \\
\hline & WATER & CUMULATIVE & & AIRBORNE & CUMULATIVE \\
\hline TANK WASTE & HAZARD & WATER & TANK WASTE & HAZARD & AIRBORNE \\
\hline CONSTITUENT(1) & INDEX & HAZARD & CONSTITUENT & INDEX & HAZARD \\
\hline TETRABROMOETHANE & $1.00 \mathrm{E}+04$ & $100.00 \%$ & DIATOMACEOUS EARTH & $5.00 \mathrm{E}-04$ & $100.00 \%$ \\
\hline TETRAHYDROFURAN & $1.00 E+04$ & $100.00 \%$ & LANTHANUM & 2.04E-04 & $100.00 \%$ \\
\hline TETRAPHENYL BORON & $1.00 E+04$ & $100.00 \%$ & STRONTIUM & $1.86 \mathrm{E}-04$ & $100.00 \%$ \\
\hline THENOYLTRIFLUOROACETONE & $1.00 E+04$ & $100.00 \%$ & ZINC & $1.26 \mathrm{E}-04$ & $100.00 \%$ \\
\hline THIOCYANATE (AMMONIUM, Na) & $1.00 \mathrm{E}+04$ & $100.00 \%$ & BISULFATE (AMMONIUM) & $9.00 \mathrm{E}-05$ & $100.00 \%$ \\
\hline THIOCYANATE (MERCURIC) & $1.00 E+04$ & $100.00 \%$ & BISULFITE & $9.00 \mathrm{E}-05$ & $100.00 \%$ \\
\hline THIOSULFATE (AMMONIUM, Na) & $1.00 E+04$ & $100.00 \%$ & BROMATE & 9.00E-05 & $100.00 \%$ \\
\hline THYMOLPTHALEIN & $1.00 \mathrm{E}+04$ & $100.00 \%$ & BROMOCRESOL PURPLE & 9.00E-05 & $100.00 \%$ \\
\hline TRHSOOCTYLAMINE & $1.00 \mathrm{E}+04$ & $100.00 \%$ & BROMONAPHTHALENE & $9.00 \mathrm{E}-05$ & $100.00 \%$ \\
\hline TRINDODECYLAMINE & $1.00 E+04$ & $100.00 \%$ & DIBUTYL PHOSPHANATE & $9.00 \mathrm{E}-05$ & $100.00 \%$ \\
\hline TRINOCTYLAMINE & $1.00 \mathrm{E}+04$ & $100.00 \%$ & DTPA & $9.00 \mathrm{E}-05$ & $100.00 \%$ \\
\hline TRIS(HYDRO.)AMINOMETHANE & $1.00 E+04$ & $100.00 \%$ & DUODECANE & 9.00E-05 & $100.00 \%$ \\
\hline UREA & $1.00 \mathrm{E}+04$ & $100.00 \%$ & FLUOROSILICATE (AMMONIUM) & $9.00 \mathrm{E}-05$ & $100.00 \%$ \\
\hline ZIRCONIUM & $2.99 \mathrm{E}+03$ & $100.00 \%$ & GLUCONATE (SODIUM) & $9.00 \mathrm{E}-05$ & $100.00 \%$ \\
\hline BERYLLIUM & $2.47 \mathrm{E}+03$ & $100.00 \%$ & GLYCEROL & $9.00 \mathrm{E}-05$ & $100.00 \%$ \\
\hline BICARBONATE & $1.67 E+03$ & $100.00 \%$ & HYDROXYLAMINE (NITRATE) & 9.00E-05 & $100.00 \%$ \\
\hline HYPOCHLORITE (SODIUM) & $1.00 \mathrm{E}+03$ & $100.00 \%$ & KEROSENE & 9.00E-05 & $100.00 \%$ \\
\hline NAPHTHYLAMINE & $1.00 \mathrm{E}+03$ & $100.00 \%$ & OPHENATROLINE & $9.00 \mathrm{E}-05$ & $100.00 \%$ \\
\hline STYRENE & $1.00 \mathrm{E}+03$ & $100.00 \%$ & PERMANGANATE (POTASSIUM) & $9.00 \mathrm{E}-05$ & $100.00 \%$ \\
\hline CERIUM & $9.44 \mathrm{E}+02$ & $100.00 \%$ & PERSULFATE (POTASSIUM) & $9.00 \mathrm{E}-05$ & $100.00 \%$ \\
\hline CESIUM & $7.50 \mathrm{E}+02$ & $100.00 \%$ & PERSULFATE (SODIUM) & $9.00 \mathrm{E}-05$ & $100.00 \%$ \\
\hline STRONTIUM & $7.24 \mathrm{E}+02$ & $100.00 \%$ & SULFITE (AMMONIUM) & $9.00 E-05$ & $100.00 \%$ \\
\hline ARSENIC & $3.30 \mathrm{E}+02$ & $100.00 \%$ & TARTRATE (SODIUM) & $9.00 \mathrm{E}-05$ & $100.00 \%$ \\
\hline TIN & $1.96 \mathrm{E}+02$ & $100.00 \%$ & TETRABROMOETHANE & $9.00 E-05$ & $100.00 \%$ \\
\hline LANTHANUM & $1.61 \mathrm{E}+02$ & $100.00 \%$ & TETRAPHENYL BORON & $9.00 E-05$ & $100.00 \%$ \\
\hline SILVER & $1.28 \mathrm{E}+02$ & $100.00 \%$ & THENOYLTRIFLUOROACETONE & 9.00E-05 & $100.00 \%$ \\
\hline RUTHENIUM & 1.17E+02 & $100.00 \%$ & THIOCYANATE (AMMONIUM, Na) & 9.00E-05 & $100.00 \%$ \\
\hline THALLIUM & $6.14 \mathrm{E}+01$ & $100.00 \%$ & THIOCYANATE (MERCURIC) & 9.00E-05 & $100.00 \%$ \\
\hline GALLIUM & $3.98 \mathrm{E}+01$ & $100.00 \%$ & THIOSULFATE (AMMONIUM, Na) & $9.00 \mathrm{E}-05$ & $100.00 \%$ \\
\hline NIOBIUM & $3.98 \mathrm{E}+01$ & $100.00 \%$ & THYMOLPTHALEIN & $9.00 \mathrm{E}-05$ & $100.00 \%$ \\
\hline TELLURIUM & $3.98 \mathrm{E}+01$ & $100.00 \%$ & TRIISOOCTYLAMINE & $9.00 \mathrm{E}-05$ & $100.00 \%$ \\
\hline COPPER & $3.93 \mathrm{E}+01$ & $100.00 \%$ & TRINDODECYLAMINE & $9.00 E-05$ & $100.00 \%$ \\
\hline BORON & $1.37 \mathrm{E}+01$ & $100.00 \%$ & TRINOCTYLAMINE & $9.00 \bar{E}-05$ & $100.00 \%$ \\
\hline
\end{tabular}


WHC-SD-WM-TI-769, Rev. 0

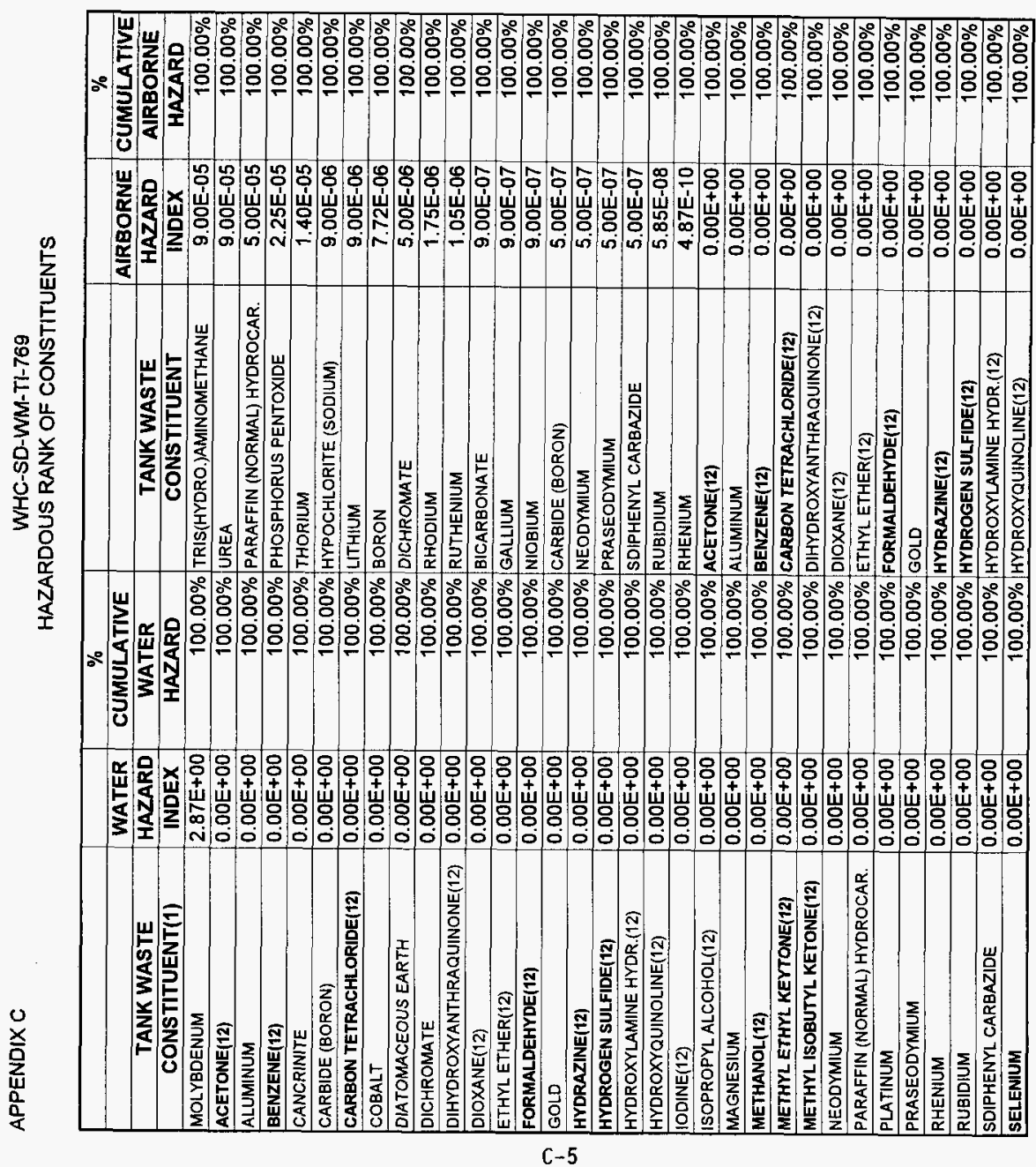


HAZARDOUS RANK OF CONSTITUENTS

\begin{tabular}{|l|c|c|c|c|c|}
\hline & & & & & $\%$ \\
\hline & WATER & CUMULATIVE & & AIRBORNE & CUMULATIVE \\
\hline \multicolumn{1}{|c|}{ TANK WASTE } & HAZARD & WATER & TANK WASTE & HAZARD & AIRBORNE \\
\hline CONSTITUENT(1) & INDEX & HAZARD & CONSTITUENT & INDEX & HAZARD \\
\hline TANTALUM & $0.00 E+00$ & $100.00 \%$ & IODINE(12) & $0.00 E+00$ & $100.00 \%$ \\
\hline THORIUM & $0.00 E+00$ & $100.00 \%$ & ISOPROPYL ALCOHOL(12) & $0.00 E+00$ & $100.00 \%$ \\
\hline TITANIUM & $0.00 E+00$ & $100.00 \%$ & METHANOL(12) & $0.00 E+00$ & $100.00 \%$ \\
\hline TRICHLOROETHANE(12) & $0.00 E+00$ & $100.00 \%$ & METHYL ETHYL KEYTONE(12) & $0.00 E+00$ & $100.00 \%$ \\
\hline TUNGSTEN & $0.00 E+00$ & $100.00 \%$ & METHYL ISOBUTYL KETONE(12) & $0.00 E+00$ & $100.00 \%$ \\
\hline VANADIUM (PENTOXIDE) & $0.00 E+00$ & $100.00 \%$ & PLATINUM & $0.00 E+00$ & $100.00 \%$ \\
\hline ZINC & $0.00 E+00$ & $100.00 \%$ & TRICHLOROETHANE(12) & $0.00 E+00$ & $100.00 \%$ \\
\hline & $3.28 E+12$ & & & $1.41 E+06$ & \\
\hline Notes: (1) Water is not included in the ranking. & & & \\
\hline
\end{tabular}

a 
WHC-SD-WM-TI-769, Rev. 0

APPENDIX D: MTCA RANK OF CHEMICALS 


\begin{tabular}{|c|c|c|c|c|c|c|c|}
\hline & TANK & & MTCA(1) & MTCA & MTCA & MTCA & $\%$ \\
\hline & SOLUBLE & & WATER & WATER & RANK OF & WATER & CUMULATIVE \\
\hline TANK WASTE & RESIDUALS & MOBILITY & STANDARD & HAZARD & TANK WASTE & HAZARD & MTCA WATER \\
\hline CONSTITUENT & $(\mathrm{kg})$ & FACTOR & (ug/L) & INDEX(2) & CONSTITUENTS & INDEX(2) & HAZARD \\
\hline ACETATE & $9.95 \mathrm{E}+03$ & $1.00 E+00$ & 1000000 & 9.95E-03 & URANIUM & $2.15 E+03$ & $46.19 \%$ \\
\hline ACETONE & $0.00 \mathrm{E}+00$ & $1.00 \mathrm{E}+00$ & 800 & $0.00 \mathrm{E}+00$ & NITRITE & $8.43 E+02$ & $64.34 \%$ \\
\hline ALUMINUM (PHOSPHIDE) & $0.00 \mathrm{E}+00$ & $3.98 \mathrm{E}-03$ & 6.4 & $0.00 \mathrm{E}+00$ & CHROMIUM (VI) & $7.26 \mathrm{E}+02$ & $79.97 \%$ \\
\hline AMMONIA (NH3) & 4.13E+04 & $1.00 E+00$ & 272000 & 1.52E-01 & NITRATE & $4.18 \mathrm{E}+02$ & $88.96 \%$ \\
\hline ANTIMONY (TRIOXIDE) & $1.00 \mathrm{E}+01$ & $1.00 \mathrm{E}+00$ & 6.4 & $1.56 \mathrm{E}+00$ & EDTA & $1.93 E+02$ & $93.13 \%$ \\
\hline ARSENIC & $4.14 \mathrm{E}-03$ & $3.98 \mathrm{E}-03$ & 4.8 & 3.44E-06 & FLUORIDE & $1.19 \mathrm{E}+02$ & $95.68 \%$ \\
\hline BARIUM & $7.79 \mathrm{E}+01$ & $1.96 \mathrm{E}-02$ & 1120 & 1.36E-03 & CHLORIDE & $5.90 \mathrm{E}+01$ & $96.95 \%$ \\
\hline BENZENE & $0.00 \mathrm{E}+00$ & $1.00 \mathrm{E}+00$ & 1.51 & $0.00 \mathrm{E}+00$ & MERCURY & $5.69 \mathrm{E}+01$ & $98.18 \%$ \\
\hline BERYLUUM & $2.48 \mathrm{E}-03$ & $3.98 \mathrm{E}-03$ & 0.0203 & 4.87E-04 & CYANIDE & $4,50 E+01$ & $99.15 \%$ \\
\hline BICARBONATE & $1.00 \mathrm{E}+01$ & $1.67 \mathrm{E}-01$ & 1000000 & 1.67E-06 & CADMIUM & $1.59 E+01$ & $99.49 \%$ \\
\hline BISULFATE (AMMONIUM) & $1.00 E+01$ & $1.00 \mathrm{E}+00$ & 1000000 & $1.00 \mathrm{E}-05$ & BUTANOL (BUTYL ALCOHOL) & $1.24 E+01$ & $99.76 \%$ \\
\hline BISULFITE & $1.00 \mathrm{E}+01$ & $1.00 \mathrm{E}+00$ & 1000000 & $1.00 \mathrm{E}-05$ & SODIUM & $6.80 \mathrm{E}+00$ & $99.90 \%$ \\
\hline BORON & $3.43 \mathrm{E}+00$ & $3.98 \mathrm{E}-03$ & 1440 & 9.49E-06 & ANTIMONY (TRIOXIDE) & $1.56 \mathrm{E}+00$ & $99.94 \%$ \\
\hline BROMATE & $1.00 \mathrm{E}+01$ & $1.00 \mathrm{E}+00$ & 1000000 & 1.00E-05 & HYDROXIDE & $1.43 \mathrm{E}+00$ & $99.97 \%$ \\
\hline BROMINE & $1.00 \mathrm{E}+01$ & $1.00 E+00$ & 1000000 & $1.00 \mathrm{E}-05$ & NICKEL & $4.24 \mathrm{E}-01$ & $99.98 \%$ \\
\hline BROMOCRESOL PURPLE & $1.00 \mathrm{E}+01$ & $1.00 E+00$ & 1000000 & $1.00 \mathrm{E}-05$ & SULFATE & $3.18 \mathrm{E}-01$ & $99.98 \%$ \\
\hline BROMONAPHTHALENE & $1.00 \mathrm{E}+01$ & $1.00 \mathrm{E}+00$ & 1000000 & $1.00 \mathrm{E}-05$ & TOC & 1.67E-01 & $99.99 \%$ \\
\hline BUTANOL (BUTYL ALCOHOL) & $1.98 \mathrm{E}+04$ & $1.00 \mathrm{E}+00$ & 1600 & $1.24 \mathrm{E}+01$ & AMMONIA (NH3) & $1.52 \mathrm{E}-01$ & $99.99 \%$ \\
\hline $\mathrm{C} 6 \mathrm{H} 507$ & $6.78 E+04$ & $1.00 \mathrm{E}+00$ & 1000000 & $6.78 \mathrm{E}-02$ & GLYCOLATE & $1.10 \mathrm{E}-01$ & $99.99 \%$ \\
\hline CADMIUM & $7.64 \mathrm{E}+02$ & 1.67E-01 & 8 & $1.59 E+01$ & HEDTA & $1.03 \mathrm{E}-01$ & $99.99 \%$ \\
\hline CALCIUM & $6.31 E+03$ & 1.67E-01 & 1000000 & 1.05E-03 & POTASSIUM & 7.07E-02 & $99.99 \%$ \\
\hline CANCRINITE & $0.00 E+00$ & $3.98 \mathrm{E}-03$ & 1000000 & $0.00 \mathrm{E}+00$ & $\mathrm{C} 6 \mathrm{H} 5 \mathrm{O} 7$ & $6.78 \mathrm{E}-02$ & $100.00 \%$ \\
\hline CARBIDE (BORON) & $0.00 \mathrm{E}+00$ & $3.98 \mathrm{E}-03$ & 1440 & $0.00 \mathrm{E}+00$ & DIBUTYUTRIBUTYL PHOSPHATE & 5.94E-02 & $100.00 \%$ \\
\hline CARBON TETRACHLORIDE(12) & $0.00 \mathrm{E}+00$ & $1.00 \mathrm{E}+00$ & 5.6 & $0.00 \mathrm{E}+00$ & THALLIUM & $5.48 \mathrm{E}-02$ & $100.00 \%$ \\
\hline CARBONATE & $4.67 \mathrm{E}+05$ & $3.98 \mathrm{E}-03$ & 1000000 & $1.86 \mathrm{E}-03$ & SUGAR & $1.00 \mathrm{E}-02$ & $100.00 \%$ \\
\hline CERIUM & $2.37 E+02$ & $3.98 \mathrm{E}-03$ & 1000000 & $9.44 \mathrm{E}-07$ & ACETATE & $9.95 \mathrm{E}-03$ & $100.00 \%$ \\
\hline CESIUM & $1.88 \mathrm{E}+02$ & $3.98 \mathrm{E}-03$ & 1000000 & $7.50 \mathrm{E}-07$ & MANGANESE & $9.48 \mathrm{E}-03$ & $100.00 \%$ \\
\hline CHLORIDE & $9.45 \mathrm{E}+04$ & $1.00 \mathrm{E}+00$ & 1600 & $5.90 \mathrm{E}+01$ & OXALATE (SODIUM) & 6.92E-03 & $100.00 \%$ \\
\hline CHROMIUM (NI) & $5.81 E+04$ & $1.00 \mathrm{E}+00$ & 80 & $7.26 \mathrm{E}+02$ & TOLUENE & $6.25 \mathrm{E}-03$ & $100.00 \%$ \\
\hline COBALT & $0.00 E+00$ & $3.98 \mathrm{E}-03$ & 1000000 & $0.00 E+00$ & PHOSPHATE & $5.00 \mathrm{E}-03$ & $100.00 \%$ \\
\hline COPPER & $9.87 \mathrm{E}+00$ & $3.98 \mathrm{E}-03$ & 592 & $6.64 \mathrm{E}-05$ & CARBONATE & $1.86 \mathrm{E}-03$ & $100.00 \%$ \\
\hline CYANIDE & $1.44 E+04$ & $1.00 E+00$ & 320 & $4.50 E+01$ & SILVER & $1.60 \mathrm{E}-03$ & $100.00 \%$ \\
\hline DIATOMACEOUS EARTH & $0.00 \mathrm{E}+00$ & $3.98 \mathrm{E}-03$ & 1000000 & $0.00 E+00$ & BARIUM & $1.36 \mathrm{E}-03$ & $100.00 \%$ \\
\hline
\end{tabular}




\begin{tabular}{|c|c|c|c|c|c|c|c|}
\hline & TANK & & MTCA(1) & MTCA & MTCA & MTCA & $\%$ \\
\hline & SOLUBLE & & WATER & WATER & RANK OF & WATER & CUMULATIVE \\
\hline TANK WASTE & RESIDUALS & MOBILITY & STANDARD & HAZARD & TANK WASTE & HAZARD & MTCA WATER \\
\hline CONSTITUENT & (kg) & FACTOR & (ug/L) & INDEX(2) & CONSTITUENTS & INDEX(2) & HAZARD \\
\hline DIBUTYL PHOSPHANATE & $1.00 E+01$ & $1.00 \mathrm{E}+00$ & 1000000 & 1.00E-05 & IRON & 1.22E-03 & $100,00 \%$ \\
\hline DIBUTYUTRIBUTYL PHOSPHATE & $5.94 E+04$ & $1.00 \mathrm{E}+00$ & 1000000 & 5.94E-02 & CALCIUM & 1.05E-03 & $100.00 \%$ \\
\hline DICHROMATE & $0.00 \mathrm{E}+00$ & $3.98 E-03$ & 1000000 & $0.00 \mathrm{E}+00$ & STYRENE & $6.25 E-04$ & $100.00 \%$ \\
\hline DIHYDROXYANTHRAQUINONE(12) & $0.00 \mathrm{E}+00$ & $1.00 \mathrm{E}+00$ & 1000000 & $0.00 E+00$ & XYLENE & $6.25 E-04$ & $100.00 \%$ \\
\hline DIOXANE(12) & $0.00 \mathrm{E}+00$ & $1.00 E+00$ & 7.95 & $0.00 \mathrm{E}+00$ & BERYLLIUM & 4.87E-04 & $100.00 \%$ \\
\hline DTPA & $1.00 \mathrm{E}+01$ & $1.00 \mathrm{E}+00$ & 1000000 & $1.00 \mathrm{E}-05$ & ETHYLENE GLYCOL & $3.13 E-04$ & $100.00 \%$ \\
\hline DUODECANE & $1.00 \mathrm{E}+01$ & $1.00 \mathrm{E}+00$ & 1000000 & 1.00E-05 & TECHNETIUM(OXIDE) & $2.20 E-04$ & $100.00 \%$ \\
\hline EDTA & $6.19 \mathrm{E}+04$ & $1.00 \mathrm{E}+00$ & 320 & $1.93 E+02$ & LEAD & 1.35E-04 & $100.00 \%$ \\
\hline ETHANOL & $1.00 \mathrm{E}+01$ & $1.00 \mathrm{E}+00$ & 1000000 & 1.00E-05 & FERROUS SULFAMATE & $1.00 E-04$ & $100.00 \%$ \\
\hline ETHYL ETHER(12) & $0.00 E+00$ & $1.00 \mathrm{E}+00$ & 1600 & $0.00 \mathrm{E}+00$ & STRONTIUM & 7.54E-05 & $100.00 \%$ \\
\hline ETHYLENE GLYCOL & $1.00 E+01$ & $1.00 \mathrm{E}+00$ & 32000 & 3.13E-04 & COPPER & 6.64E-05 & $100.00 \%$ \\
\hline FERROUS SULFAMATE & $1.00 \mathrm{E}+02$ & $1.00 \mathrm{E}+00$ & 1000000 & $1.00 \mathrm{E}-04$ & MOLYBDENUM & 3.59E-05 & $100.00 \%$ \\
\hline FLUORIDE & $1.14 \mathrm{E}+05$ & $1.00 \mathrm{E}+00$ & 960 & 1.19E+02 & TIN & 2.04E-05 & $100.00 \%$ \\
\hline FLUOROSILICATE (AMMONIUM) & $1.00 \mathrm{E}+01$ & $1.00 \mathrm{E}+00$ & 1000000 & $1.00 \mathrm{E}-05$ & RHODIUM & 1.94E-05 & $100.00 \%$ \\
\hline FORMALDEHYDE(12) & $0.00 \mathrm{E}+00$ & $1.00 \mathrm{E}+00$ & 1.46 & $0.00 E+00$ & SILICON (SILICATE) & $1.56 E-05$ & $100.00 \%$ \\
\hline GALLIUM & $1.00 \mathrm{E}+01$ & $3.98 \mathrm{E}-03$ & 1000000 & $3.98 \mathrm{E}-08$ & BISULFATE (AMMONIUM) & $1.00 E-05$ & $100.00 \%$ \\
\hline GLUCONATE (SODIUM) & $1.00 E+01$ & $1.00 \mathrm{E}+00$ & 1000000 & 1.00E-05 & BISULFITE & $1.00 \mathrm{E}-05$ & $100.00 \%$ \\
\hline GLYCEROL & $1.00 \mathrm{E}+01$ & $1.00 \mathrm{E}+00$ & 1000000 & 1.00E-05 & BROMATE & $1.00 \mathrm{E}-05$ & $100.00 \%$ \\
\hline GLYCOLATE & $1.10 \mathrm{E}+05$ & $1.00 \mathrm{E}+00$ & 1000000 & $1.10 \mathrm{E}-01$ & BROMINE & 1.00E-05 & $100.00 \%$ \\
\hline GOLD & $0.00 \mathrm{E}+00$ & 3.98E-03 & 1000000 & $0.00 \mathrm{E}+00$ & BROMOCRESOL PURPLE & 1.00E-05 & $100.00 \%$ \\
\hline HEDTA & $1,03 \mathrm{E}+05$ & $1.00 \mathrm{E}+00$ & 1000000 & 1.03E-01 & BROMONAPHTHALENE & 1.00E-05 & $100.00 \%$ \\
\hline HYDRAZINE(12) & $0.00 \mathrm{E}+00$ & $1.00 \mathrm{E}+00$ & 0.0146 & $0.00 \mathrm{E}+00$ & DIBUTYL PHOSPHANATE & 1.00E-05 & $100.00 \%$ \\
\hline HYDROGEN SULFIDE(12) & $0.00 \mathrm{E}+00$ & $1.00 \mathrm{E}+00$ & 24 & $0.00 E+00$ & DTPA & 1.00E-05 & $100.00 \%$ \\
\hline HYDROXIDE & $1.43 \mathrm{E}+06$ & $1.00 \mathrm{E}+00$ & 1000000 & $1.43 E+00$ & DUODECANE & 1.00E-05 & $100.00 \%$ \\
\hline HYDROXYLAMINE (NITRATE) & $1.00 \mathrm{E}+01$ & $1.00 \mathrm{E}+00$ & 1000000 & 1.00E-05 & ETHANOL & 1.00E-05 & $100.00 \%$ \\
\hline HYDROXYLAMINE HYDR.(12) & $0.00 \mathrm{E}+00$ & $1.00 \mathrm{E}+00$ & 1000000 & $0.00 \mathrm{E}+00$ & FLUOROSILICATE (AMMONIUM) & 1.00E-05 & $100.00 \%$ \\
\hline HYDROXYQUINOLINE(12) & $0.00 \mathrm{E}+00$ & $1.00 \mathrm{E}+00$ & 1000000 & $0.00 \mathrm{E}+00$ & GLUCONATE (SODIUM) & 1.00E-05 & $100.00 \%$ \\
\hline HYPOCHLORITE (SODIUM) & $1.00 \mathrm{E}+00$ & $1.00 E+00$ & 1000000 & 1.00E-06 & GLYCEROL & 1.00E-05 & $100.00 \%$ \\
\hline IODINE(12) & $0.00 \mathrm{E}+00$ & $1.00 \mathrm{E}+00$ & 1000000 & $0.00 \mathrm{E}+00$ & HYDROXYLAMINE (NITRATE) & 1.00E-05 & $100.00 \%$ \\
\hline IRON & $7.32 \mathrm{E}+03$ & $1.67 \mathrm{E}-01$ & 1000000 & $1.22 \mathrm{E}-03$ & ISOAMYL ACETATE & 1.00E-05 & $100.00 \%$ \\
\hline ISOAMYL ACETATE & $1.00 \mathrm{E}+01$ & $1.00 \mathrm{E}+00$ & 1000000 & 1.00E-05 & KEROSENE & $1.00 \mathrm{E}-05$ & $100.00 \%$ \\
\hline ISOPROPYL ALCOHOL(12) & $0.00 E+00$ & $1.00 \mathrm{E}+00$ & 1000000 & $0.00 \mathrm{E}+00$ & LITHIUM & 1.00E-05 & $100.00 \%$ \\
\hline KEROSENE & $1.00 \mathrm{E}+01$ & $1.00 \mathrm{E}+00$ & 1000000 & $1.00 \mathrm{E}-05$ & MORPHOLINE & 1.00E-05 & $100.00 \%$ \\
\hline
\end{tabular}




\begin{tabular}{|c|c|c|c|c|c|c|c|}
\hline & TANK & & MTCA(1) & MTCA & MTCA & MTCA & $\%$ \\
\hline & SOLUBLE & & WATER & WATER & RANK OF & WATER & CUMULATIVE \\
\hline TANK WASTE & RESIDUALS & MOBILITY & STANDARD & HAZARD & TANK WASTE & HAZARD & MTCA WATER \\
\hline CONSTITUENT & (kg) & \begin{tabular}{|l|} 
FACTOR \\
\end{tabular} & (ug/L) & INDEX(2) & CONSTITUENTS & INDEX(2) & HAZARD \\
\hline LANTHANUM & $4.04 \mathrm{E}+01$ & $3.98 \mathrm{E}-03$ & 1000000 & $1.61 \mathrm{E}-07$ & OPHENATROLINE & $1.00 \mathrm{E}-05$ & $100.00 \%$ \\
\hline LEAD & $6.88 \mathrm{E}+03$ & $1.96 \mathrm{E}-02$ & 1000000 & $1.35 \mathrm{E}-04$ & PERMANGANATE (POTASSIUM) & 1.00E-05 & $100.00 \%$ \\
\hline LITHIUM & $1.00 \mathrm{E}+01$ & $1.00 E+00$ & 1000000 & $1.00 \mathrm{E}-05$ & PERSULFATE (POTASSIUM) & $1.00 \mathrm{E}-05$ & $100.00 \%$ \\
\hline MAGNESIUM & $0.00 \mathrm{E}+00$ & $3.98 \mathrm{E}-03$ & 1000000 & $0.00 \mathrm{E}+00$ & PERSULFATE (SODIUM) & 1.00E-05 & $100.00 \%$ \\
\hline MANGANESE & $1.08 E+03$ & $1.96 \mathrm{E}-02$ & 2240 & $9.48 E-03$ & PHOSPHORUS PENTOXIDE & $1.00 \mathrm{E}-05$ & $100.00 \%$ \\
\hline MERCURY & $2.73 \mathrm{E}+02$ & $1.00 \mathrm{E}+00$ & 4.8 & $5.69 E+01$ & SULFITE (AMMONIUM) & 1.00E-05 & $100.00 \%$ \\
\hline METHANOL(12) & $0.00 \mathrm{E}+00$ & $1.00 \mathrm{E}+00$ & 4000 & $0.00 \mathrm{E}+00$ & TARTRATE (SODIUM) & $1.00 \mathrm{E}-05$ & $100.00 \%$ \\
\hline METHYL ETHYL KEYTONE(12) & $0.00 \mathrm{E}+00$ & $1.00 \mathrm{E}+00$ & 4800 & $0.00 \mathrm{E}+00$ & TETRABROMOETHANE & $1.00 \mathrm{E}-05$ & $100.00 \%$ \\
\hline METHYL ISOBUTYL KETONE(12) & $0.00 \mathrm{E}+00$ & $1.00 \mathrm{E}+00$ & 640 & $0.00 \mathrm{E}+00$ & TETRAHYDROFURAN & 1.00E-05 & $100.00 \%$ \\
\hline MOLYBDENUM & $7.21 E-01$ & $3.98 \mathrm{E}-03$ & 80 & $3.59 \mathrm{E}-05$ & TETRAPHENYL BORON & $1.00 \mathrm{E}-05$ & $100.00 \%$ \\
\hline MORPHOLINE & $1.00 \mathrm{E}+01$ & $1.00 \mathrm{E}+00$ & 1000000 & $1.00 \mathrm{E}-05$ & THENOYLTRIFLUOROACETONE & 1.00E-05 & $100.00 \%$ \\
\hline NAPHTHYLAMINE & $1.00 \mathrm{E}+00$ & $1.00 E+00$ & 1000000 & $1.00 \mathrm{E}-06$ & THIOCYANATE (AMMONIUM, Na) & $1.00 \mathrm{E}-05$ & $100.00 \%$ \\
\hline NEODYMIUM & $0.00 \mathrm{E}+00$ & $3.98 \mathrm{E}-03$ & 1000000 & $0.00 E+00$ & THIOCYANATE (MERCURIC) & $1.00 \mathrm{E}-05$ & $100.00 \%$ \\
\hline NICKEL & $8.13 E+02$ & 1.67E-01 & 320 & 4.24E-01 & THIOSULFATE (AMMONIUM, Na) & 1.00E-05 & $100.00 \%$ \\
\hline NIOBIUM & $1.00 \mathrm{E}+01$ & $3.98 E-03$ & 1000000 & $3.98 \mathrm{E}-08$ & THYMOLPTHALEIN & $1.00 \mathrm{E}-05$ & $100.00 \%$ \\
\hline NITRATE & $1.07 \mathrm{E}+07$ & $1.00 \mathrm{E}+00$ & 25600 & $4.18 \mathrm{E}+02$ & TRIISOOCTYLAMINE & $1.00 \mathrm{E}-05$ & $100.00 \%$ \\
\hline NITRITE & $1.35 \mathrm{E}+06$ & $1.00 E+00$ & 1600 & $8.43 E+02$ & TRINDODECYLAMINE & $1.00 \mathrm{E}-05$ & $100.00 \%$ \\
\hline OPHENATROLINE & $1.00 \mathrm{E}+01$ & $1.00 \mathrm{E}+00$ & 1000000 & $1.00 \mathrm{E}-05$ & TRINOCTYLAMINE & $1.00 \mathrm{E}-05$ & $100.00 \%$ \\
\hline OXALATE (SODIUM) & $6.92 \mathrm{E}+03$ & $1.00 \mathrm{E}+00$ & 1000000 & $6.92 \mathrm{E}-03$ & TRIS(HYDRO.)AMINOMETHANE & $1.00 \mathrm{E}-05$ & $100.00 \%$ \\
\hline PARAFFIN (NORMAL) HYDROCAR. & $0.00 \mathrm{E}+00$ & $3.98 \mathrm{E}-03$ & 1000000 & $0.00 \mathrm{E}+00$ & UREA & $1.00 \mathrm{E}-05$ & $100.00 \%$ \\
\hline PERMANGANATE (POTASSIUM) & $1.00 \mathrm{E}+01$ & $1.00 \mathrm{E}+00$ & 1000000 & $1.00 \mathrm{E}-05$ & BORON & $9.49 \mathrm{E}-06$ & $100.00 \%$ \\
\hline PERSULFATE (POTASSIUM) & $1.00 \mathrm{E}+01$ & $1.00 \mathrm{E}+00$ & 1000000 & $1.00 \mathrm{E}-05$ & ARSENIC & $3.44 \mathrm{E}-06$ & $100.00 \%$ \\
\hline PERSULFATE (SODIUM) & $1.00 \mathrm{E}+01$ & $1.00 \mathrm{E}+00$ & 1000000 & 1.00E-05 & ZIRCONIUM & 2.99E-06 & $100.00 \%$ \\
\hline PHOSPHATE & $2.55 \mathrm{E}+05$ & $1.96 \mathrm{E}-02$ & 1000000 & $5.00 \mathrm{E}-03$ & BICARBONATE & $1.67 \mathrm{E}-06$ & $100.00 \%$ \\
\hline PHOSPHORUS PENTOXIDE & $1.00 \mathrm{E}+01$ & $1.00 \mathrm{E}+00$ & 1000000 & $1.00 \mathrm{E}-05$ & HYPOCHLORITE (SODIUM) & 1.00E-06 & $100.00 \%$ \\
\hline PLATINUM & $0.00 \mathrm{E}+00$ & $3.98 \mathrm{E}-03$ & 1000000 & $0.00 \mathrm{E}+00$ & NAPHTHYLAMINE & 1.00E-06 & $100.00 \%$ \\
\hline POTASSIUM & $7.07 \mathrm{E}+04$ & $1.00 \mathrm{E}+00$ & 1000000 & \begin{tabular}{|l|}
$7.07 \mathrm{E}-02$ \\
\end{tabular} & CERIUM & 9.44E-07 & $100.00 \%$ \\
\hline PRASEODYMIUM & $0.00 \mathrm{E}+00$ & 3.98E-03 & 1000000 & $0.00 \mathrm{E}+00$ & CESIUM & $7.50 \mathrm{E}-07$ & $100.00 \%$ \\
\hline RHENIUM & $0.00 \mathrm{E}+00$ & $3.98 \mathrm{E}-03$ & 1000000 & $0.00 \mathrm{E}+00$ & LANTHANUM & 1.61E-07 & $100.00 \%$ \\
\hline RHODIUM & $1.94 \mathrm{E}+01$ & $1.00 \mathrm{E}+00$ & 1000000 & 1.94E-05 & RUTHENIUM & 1.17E-07 & $100.00 \%$ \\
\hline RUBIDIUM & $0.00 \mathrm{E}+00$ & 3.98 E-03 & 1000000 & $0.00 \mathrm{E}+00$ & GALLIUM & $3.98 E-08$ & $100.00 \%$ \\
\hline RUTHENIUM & 1.17E-01 & $1.00 \mathrm{E}+00$ & 1000000 & 1.17E-07 & NIOBIUM & $3.98 \mathrm{E}-08$ & $100.00 \%$ \\
\hline SDIPHENYL CARBAZIDE & $0.00 \mathrm{E}+00$ & $1.00 \mathrm{E}+00$ & 1000000 & $0.00 \mathrm{E}+00$ & TELLURIUM & $3.98 \mathrm{E}-08$ & $100.00 \%$ \\
\hline
\end{tabular}




\begin{tabular}{|c|c|c|c|c|c|c|c|}
\hline & TANK & & MTCA(1) & MTCA & MTCA & MTCA & $\%$ \\
\hline & SOLUBLE & & WATER & WATER & RANK OF & WATER & CUMULATIVE \\
\hline TANK WASTE & RESIDUALS & MOBILITY & STANDARD & HAZARD & TANK WASTE & HAZARD & MTCA WATER \\
\hline CONSTITUENT & (kg) & FACTOR & (ug/L) & INDEX(2) & CONSTITUENTS & INDEX(2) & HAZARD \\
\hline SELENIUM & $0.00 \mathrm{E}+00$ & $1.00 \mathrm{E}+00$ & 80 & $0.00 \mathrm{E}+00$ & ACETONE & $0.00 \mathrm{E}+00$ & $100.00 \%$ \\
\hline SILICON (SILICATE) & $3.92 \mathrm{E}+03$ & $3.98 \mathrm{E}-03$ & 1000000 & $1.56 \mathrm{E}-05$ & ALUMINUM (PHOSPHIDE) & $0.00 \mathrm{E}+00$ & $100.00 \%$ \\
\hline SILVER & $3.20 \mathrm{E}+01$ & $3.98 \mathrm{E}-03$ & 80 & $1.60 \mathrm{E}-03$ & BENZENE & $0.00 \mathrm{E}+00$ & $100.00 \%$ \\
\hline SODIUM & $6.80 \mathrm{E}+06$ & $1.00 \mathrm{E}+00$ & 1000000 & $6.80 \mathrm{E}+00$ & CANCRINITE & $0.00 \mathrm{E}+00$ & $100.00 \%$ \\
\hline STRONTIUM & $3.69 E+01$ & $1.96 \mathrm{E}-02$ & 9600 & 7.54E-05 & CARBIDE (BORON) & $0.00 \mathrm{E}+00$ & $100.00 \%$ \\
\hline STYRENE & $1.00 \mathrm{E}+00$ & $1.00 \mathrm{E}+00$ & 1600 & $6.25 \mathrm{E}-04$ & CARBON TETRACHLORIDE(12) & $0.00 E+00$ & $100.00 \%$ \\
\hline SUGAR & $1.00 E+04$ & $1.00 \mathrm{E}+00$ & 1000000 & $1.00 \mathrm{E}-02$ & COBALT & $0.00 \mathrm{E}+00$ & $100.00 \%$ \\
\hline SULFATE & $3.18 \mathrm{E}+05$ & $1.00 \mathrm{E}+00$ & 1000000 & $3.18 \mathrm{E}-01$ & DIATOMACEOUS EARTH & $0.00 \mathrm{E}+00$ & $100.00 \%$ \\
\hline SULFITE (AMMONIUM) & $1.00 \mathrm{E}+01$ & $1.00 E+00$ & 1000000 & $1.00 \mathrm{E}-05$ & DICHROMATE & $0.00 \mathrm{E}+00$ & $100.00 \%$ \\
\hline TANTALUM & $0.00 \mathrm{E}+00$ & $3.98 \mathrm{E}-03$ & 1000000 & $0.00 \mathrm{E}+00$ & DIHYDROXYANTHRAQUINONE(12) & $0.00 E+00$ & $100.00 \%$ \\
\hline TARTRATE (SODIUM) & $1.00 E+01$ & $1.00 \mathrm{E}+00$ & 1000000 & 1.00E-05 & DIOXANE(12) & $0.00 \mathrm{E}+00$ & $100.00 \%$ \\
\hline TECHNETIUM(OXIDE) & $2.20 \mathrm{E}+02$ & $1.00 \mathrm{E}+00$ & 1000000 & $2.20 \mathrm{E}-04$ & ETHYL ETHER(12) & $0.00 \mathrm{E}+00$ & $100.00 \%$ \\
\hline TELLURIUM & $1.00 E+01$ & $3.98 \mathrm{E}-03$ & 1000000 & $3.98 \mathrm{E}-08$ & FORMALDEHYDE(12) & $0.00 \mathrm{E}+00$ & $100.00 \%$ \\
\hline TETRABROMOETHANE & $1.00 \mathrm{E}+01$ & $1.00 \mathrm{E}+00$ & 1000000 & $1.00 \mathrm{E}-05$ & GOLD & $0.00 E+00$ & $100.00 \%$ \\
\hline TETRAHYDROFURAN & $1.00 \mathrm{E}+01$ & $1.00 \mathrm{E}+00$ & 1000000 & $1.00 \mathrm{E}-05$ & HYDRAZINE(12) & $0.00 \mathrm{E}+00$ & $100.00 \%$ \\
\hline TETRAPHENYL BORON & $1.00 \mathrm{E}+01$ & $1.00 \mathrm{E}+00$ & 1000000 & $1.00 \mathrm{E}-05$ & HYDROGEN SULFIDE(12) & $0.00 \mathrm{E}+00$ & $100.00 \%$ \\
\hline THALLIUM & $1.54 \mathrm{E}+01$ & $3.98 \mathrm{E}-03$ & 1.12 & $5.48 \mathrm{E}-02$ & HYDROXYLAMINE HYDR.(12) & $0.00 \mathrm{E}+00$ & $100.00 \%$ \\
\hline THENOYLTRIFLUOROACETONE & $1.00 \mathrm{E}+01$ & $1.00 \mathrm{E}+00$ & 1000000 & $1.00 \mathrm{E}-05$ & HYDROXYQUINOLINE(12) & $0.00 \mathrm{E}+00$ & $100.00 \%$ \\
\hline THIOCYANATE (AMMONIUM, Na) & $1.00 \mathrm{E}+01$ & $1.00 \mathrm{E}+00$ & 1000000 & $1.00 \mathrm{E}-05$ & IODINE(12) & $0.00 \mathrm{E}+00$ & $100.00 \%$ \\
\hline THIOCYANATE (MERCURIC) & $1.00 \mathrm{E}+01$ & $1.00 \mathrm{E}+00$ & 1000000 & $1.00 \mathrm{E}-05$ & ISOPROPYL ALCOHOL(12) & $0.00 \mathrm{E}+00$ & $100.00 \%$ \\
\hline THIOSULFATE (AMMONIUM, Na) & $1.00 \mathrm{E}+01$ & $1.00 \mathrm{E}+00$ & 1000000 & $1.00 \mathrm{E}-05$ & MAGNESIUM & $0.00 \mathrm{E}+00$ & $100.00 \%$ \\
\hline THORIUM & $0.00 \mathrm{E}+00$ & $1.96 \mathrm{E}-02$ & 1000000 & $0.00 \mathrm{E}+00$ & METHANOL(12) & $0.00 \mathrm{E}+00$ & $100.00 \%$ \\
\hline THYMOLPTHALEIN & $1.00 \mathrm{E}+01$ & $1.00 \mathrm{E}+00$ & 1000000 & 1.00E-05 & METHYL ETHYL KEYTONE(12) & $0.00 E+00$ & $100.00 \%$ \\
\hline TIN & $1.00 \mathrm{E}+01$ & $1.96 \mathrm{E}-02$ & 9600 & 2.04E-05 & METHYL ISOBUTYL KETONE(12) & $0.00 E+00$ & $100.00 \%$ \\
\hline TITANIUM & $0.00 \mathrm{E}+00$ & $3.98 \mathrm{E}-03$ & 1000000 & $0.00 E+00$ & NEODYMIUM & $0.00 E+00$ & $100.00 \%$ \\
\hline TOC & $1.67 E+05$ & $1.00 \mathrm{E}+00$ & 1000000 & 1.67E-01 & PARAFFIN (NORMAL) HYDROCAR. & $0.00 \mathrm{E}+00$ & $100.00 \%$ \\
\hline TOLUENE & $1.00 E+01$ & $1.00 \mathrm{E}+00$ & 1600 & $6.25 E-03$ & PLATINUM & $0.00 \mathrm{E}+00$ & $100.00 \%$ \\
\hline TRICHLOROETHANE(12) & $0.00 \mathrm{E}+00$ & $1.00 \mathrm{E}+00$ & 7200 & $0.00 \mathrm{E}+00$ & PRASEODYMIUM & $0.00 \mathrm{E}+00$ & $100.00 \%$ \\
\hline TRIISOOCTYLAMINE & $1.00 \mathrm{E}+01$ & $1.00 \mathrm{E}+00$ & 1000000 & 1.00E-05 & RHENIUM & $0.00 \mathrm{E}+00$ & $100.00 \%$ \\
\hline TRINDODECYLAMINE & $1.00 \mathrm{E}+01$ & $1.00 \mathrm{E}+00$ & 1000000 & 1.00E-05 & RUBIDIUM & $0.00 \mathrm{E}+00$ & $100.00 \%$ \\
\hline TRINOCTYLAMINE & $1.00 \mathrm{E}+01$ & $1.00 E+00$ & 1000000 & $1.00 \mathrm{E}-05$ & SDIPHENYL CARBAZIDE & $0.00 \mathrm{E}+00$ & $100.00 \%$ \\
\hline TRIS(HYDRO,)AMINOMETHANE & $1.00 \mathrm{E}+01$ & $1.00 \mathrm{E}+00$ & 1000000 & $1.00 \mathrm{E}-05$ & SELENIUM & $0.00 \mathrm{E}+00$ & $100.00 \%$ \\
\hline TUNGSTEN & $0.00 \mathrm{E}+00$ & $1.00 \mathrm{E}+00$ & 1000000 & $0.00 \mathrm{E}+00$ & TANTALUM & $0.00 \mathrm{E}+00$ & $100.00 \%$ \\
\hline
\end{tabular}




\begin{tabular}{|c|c|c|c|c|c|c|c|}
\hline & TANK & & MTCA(1) & MTCA & MTCA & MTCA & $\%$ \\
\hline & SOLUBLE & & WATER & WATER & RANK OF & WATER & CUMULATIVE \\
\hline TANK WASTE & RESIDUALS & MOBILITY & STANDARD & HAZARD & TANK WASTE & HAZARD & MTCA WATER \\
\hline CONSTITUENT & $(\mathrm{kg})$ & FACTOR & (ug/L) & INDEX(2) & CONSTITUENTS & INDEX(2) & HAZARD \\
\hline URANIUM & $1.03 E+05$ & $1.00 \mathrm{E}+00$ & 48 & $2.15 E+03$ & THORIUM & $0.00 \mathrm{E}+00$ & $100.00 \%$ \\
\hline UREA & $1.00 \mathrm{E}+01$ & $1.00 \mathrm{E}+00$ & 1000000 & $1.00 \mathrm{E}-05$ & TITANIUM & $0.00 \mathrm{E}+00$ & $100.00 \%$ \\
\hline VANADIUM (PENTOXIDE) & $0.00 \mathrm{E}+00$ & $3.98 \mathrm{E}-03$ & 144 & $0.00 \mathrm{E}+00$ & TRICHLOROETHANE(12) & $0.00 \mathrm{E}+00$ & $100.00 \%$ \\
\hline XYLENE & $1.00 \mathrm{E}+01$ & $1.00 \mathrm{E}+00$ & 16000 & $6.25 \mathrm{E}-04$ & TUNGSTEN & $0.00 \mathrm{E}+00$ & $100.00 \%$ \\
\hline ZINC & $0.00 \mathrm{E}+00$ & $3.98 \mathrm{E}-03$ & 4800 & $0.00 E+00$ & VANADIUM (PENTOXIDE) & $0.00 \mathrm{E}+00$ & $100.00 \%$ \\
\hline \multirow[t]{2}{*}{ ZIRCONIUM } & $7.50 \mathrm{E}+02$ & $3.98 \mathrm{E}-03$ & 1000000 & 2.99E-06 & ZINC & $0.00 \mathrm{E}+00$ & $100.00 \%$ \\
\hline & & & & & & $4.65 E+03$ & \\
\hline $\begin{array}{l}\text { 1. These are groundw } \\
\text { a value of } 1.0 \mathrm{E}+6 \\
\text { 2. The hazard index }\end{array}$ & entory time & mobilit & $\begin{array}{l}\text { Not } \\
\text { s Control }\end{array}$ & & & & \\
\hline
\end{tabular}

o 


\begin{tabular}{|c|c|c|c|c|c|}
\hline \multicolumn{6}{|c|}{ DISTRIBUTION SHEET } \\
\hline \multirow{2}{*}{$\begin{array}{l}\text { To } \\
\text { Distribution }\end{array}$} & \multirow{2}{*}{\multicolumn{3}{|c|}{$\begin{array}{l}\text { From } \\
\text { Process Technology } 73510\end{array}$}} & \multicolumn{2}{|c|}{ Page 1 of 1} \\
\hline & & & & \multicolumn{2}{|c|}{ Date $8 / 30 / 96$} \\
\hline \multicolumn{4}{|c|}{ Project Title/Work Order } & \multicolumn{2}{|c|}{ EDT No. 615774} \\
\hline Predominant Chemicals & Site Waste Ta & $\mathrm{ks} / \mathrm{D2086}$ & & \multicolumn{2}{|c|}{ ECN No. } \\
\hline Name & MSIN & $\begin{array}{c}\text { Text } \\
\text { With All } \\
\text { Attach. }\end{array}$ & Text Only & $\begin{array}{l}\text { Attach./ } \\
\text { Appendix } \\
\text { Only }\end{array}$ & $\begin{array}{l}\text { EDT/ECN } \\
\text { Only }\end{array}$ \\
\hline $\begin{array}{l}\text { A. L. Boldt } \\
\text { G. F. Boothe (20) } \\
\text { L. E. Borneman } \\
\text { E. A. Fredenburg (5) } \\
\text { M. E. Hevland } \\
\text { M. N. Islam } \\
\text { M. J. Klem } \\
\text { M. J. Kupfer (5) } \\
\text { G. A. Meyer } \\
\text { C. A. Petersen } \\
\text { D. E. Place } \\
\text { J. C. Sonnichsen } \\
\text { W. E. Toebe } \\
\text { D. J. Washenfelder } \\
\text { C. D. West } \\
\text { Central Files }\end{array}$ & $\begin{array}{l}H 5-49 \\
H 5-27 \\
\text { R1-90 } \\
H 5-61 \\
\text { P7-02 } \\
\text { R3-08 } \\
H 5-27 \\
H 5-27 \\
S 2-48 \\
H 5-27 \\
H 5-27 \\
H 6-24 \\
H 6-22 \\
H 5-27 \\
S 7-53 \\
A 3-88 \\
\end{array}$ & $\begin{array}{l}\mathrm{x} \\
\mathrm{x} \\
\mathrm{x} \\
\mathrm{x} \\
\mathrm{x} \\
\mathrm{x} \\
\mathrm{x} \\
\mathrm{x} \\
\mathrm{x} \\
\mathrm{x} \\
\mathrm{x} \\
\mathrm{x} \\
\mathrm{x} \\
\mathrm{x} \\
\mathrm{x} \\
\mathrm{x}\end{array}$ & & & \\
\hline
\end{tabular}

